\title{
A Highly Stereocontrolled Total Synthesis of (-)-Neodysiherbaine A
}

Keisuke Takahashi, Takashi Matsumura, Graeme R. M. Corbin, Jun Ishihara, and Susumi Hatakeyama*

Graduate School of Biomedical Sciences, Nagasaki University, Nagasaki 852-8521, Japan

\section{Supporting Information}

Table of Contents

Page

General Experimental

S2

Synthesis of compound $\mathbf{1 4}$ from 9

$\mathrm{S} 2$

${ }^{1} \mathrm{H}$ and ${ }^{13} \mathrm{C}$ NMR spectra for $\mathbf{1 1}$

S4

${ }^{1} \mathrm{H}$ and ${ }^{13} \mathrm{C}$ NMR spectra for $\mathbf{1 2}$

S5

${ }^{1} \mathrm{H}$ and ${ }^{13} \mathrm{C}$ NMR spectra for $\mathbf{1 4}$

S6

${ }^{1} \mathrm{H}$ and ${ }^{13} \mathrm{C}$ NMR spectra for $\mathbf{1 5}$

$\mathrm{S} 7$

${ }^{1} \mathrm{H}$ and ${ }^{13} \mathrm{C}$ NMR spectra for $\mathbf{1 6}$

S8

${ }^{1} \mathrm{H}$ and ${ }^{13} \mathrm{C}$ NMR spectra for 17

S9

${ }^{1} \mathrm{H}$ and ${ }^{13} \mathrm{C}$ NMR spectra for $\mathbf{1 8}$

S10

${ }^{1} \mathrm{H}$ and ${ }^{13} \mathrm{C}$ NMR spectra for 20

$\mathrm{S} 11$

${ }^{1} \mathrm{H}$ and ${ }^{13} \mathrm{C}$ NMR spectra for $\mathbf{2 1}$

S12

${ }^{1} \mathrm{H}$ and ${ }^{13} \mathrm{C}$ NMR spectra for 22

$\mathrm{S} 13$

${ }^{1} \mathrm{H}$ and ${ }^{13} \mathrm{C}$ NMR spectra for $\mathbf{2 5}$

S14

${ }^{1} \mathrm{H}$ and ${ }^{13} \mathrm{C}$ NMR spectra for $\mathbf{2 6}$

S15

${ }^{1} \mathrm{H}$ and ${ }^{13} \mathrm{C}$ NMR spectra for 27

S16

${ }^{1} \mathrm{H}$ and ${ }^{13} \mathrm{C}$ NMR spectra for 29

S17

H-H COSY and NOESY spectra for 29

S18

${ }^{1} \mathrm{H}$ and ${ }^{13} \mathrm{C}$ NMR spectra for neodysiherbaine $\mathrm{A}(\mathbf{2})$

S19 
General Experimental. All reactions were performed under argon atmosphere, except for DDQ oxidation of 19 and $\mathrm{NaIO}_{4}$ oxidation of 28. All extracts were dried over $\mathrm{MgSO}_{4}$ and concentrated by rotary evaporation below $30{ }^{\circ} \mathrm{C}$ at 25 Toor unless otherwise noted. Commercial reagents and solvents were used as supplied with following exceptions. Dichloromethane $\left(\mathrm{CH}_{2} \mathrm{Cl}_{2}\right)$, triethylamine $\left(\mathrm{Et}_{3} \mathrm{~N}\right)$, dimethyl sulfoxide (DMSO), $\mathrm{N}, \mathrm{N}$ dimethylformamide (DMF), $\quad N, N$-dimethylacetoamide $\quad$ (DMA), $\quad N, N, N, N^{\prime}, N^{\prime}, N^{\prime}-$ hexamethylphosphoramide (HMPA), benzene, acetonitrile (MeCN) were distilled from $\mathrm{CaH}_{2}$. Methanol $(\mathrm{MeOH})$ was distilled from sodium. Analytical thin-layer chromatography (TLC) was performed using glass-packed silica gel plates $(0.2 \mathrm{~mm}$ thickness $)$. Column chromatography was performed using silica gel (particle size 100-210 $\mu$ ). Optical rotations were recorded on a digital polarimeter at ambient temperature. Infrared spectra were measured on a Fourier transform infrared spectrometer. ${ }^{1} \mathrm{H}$ NMR $(400$ and $500 \mathrm{MHz})$ and ${ }^{13} \mathrm{C}$ NMR (100 MHz) spectra were measured using $\mathrm{CDCl}_{3}, \mathrm{CD}_{3} \mathrm{OD}$, or $\mathrm{D}_{2} \mathrm{O}$ as solvent, and chemical shifts are reported as $\delta$ values in ppm based on internal $\mathrm{CHCl}_{3}\left(7.26 \mathrm{ppm},{ }^{1} \mathrm{H} ; 77.0\right.$ ppm, $\left.{ }^{13} \mathrm{C}\right), \mathrm{H}_{2} \mathrm{O}\left(4.65 \mathrm{ppm},{ }^{1} \mathrm{H}\right)$, or $\mathrm{MeOH}\left(49.9 \mathrm{ppm},{ }^{13} \mathrm{C}\right)$. HRMS spectra were taken in EI or FAB mode.

(3aR,6R,7R,7aR)-Tetrahydro-(7-(tert-butyldimethylsilyl)oxy-2,2-dimethyl-3aH[1,3]dioxolo[4,5-c]pyran-6-yl)methanol (14). To a stirred solution of tri- $O$-acetyl-D-glucal (9) $(71 \mathrm{~g}, 261 \mathrm{mmol})$ in $\mathrm{CH}_{2} \mathrm{Cl}_{2}(600 \mathrm{ml})$ at $0{ }^{\circ} \mathrm{C}$ were added triethylsilane $(73 \mathrm{~g}, 522 \mathrm{mmol})$ and $\mathrm{BF}_{3} \cdot \mathrm{OEt}_{2}(54 \mathrm{~g}, 470 \mathrm{mmol})$. After stirring at $0{ }^{\circ} \mathrm{C}$ for $2 \mathrm{~h}$, the reaction was quenched with saturated $\mathrm{NaHCO}_{3}(300 \mathrm{ml})$. The reaction mixture was extracted with $\mathrm{CHCl}_{3}$, washed with brine and concentrated to give $((2 R, 3 S)$-3,6-dihydro-3-acetoxy-2H-pyran-2-yl)methyl acetate $(60 \mathrm{~g})$ as a colorless oil, which was used for the next reaction without purification.

Crude $((2 R, 3 S)$-3,6-dihydro-3-acetoxy-2H-pyran-2-yl)methyl acetate $(60 \mathrm{~g})$ was dissolved into $\mathrm{MeOH}(300 \mathrm{ml})$ and $\mathrm{NaOMe}(4.2 \mathrm{~g}, 78.3 \mathrm{mmol})$ was added. After being stirred for $2 \mathrm{~h}$ at room temperature, the reaction mixture was neutralized with Dowex 50, filtrated, and evaporated to give $\mathbf{1 0}(40 \mathrm{~g})$ as a colorless solid, which was used for the next reaction without purification.

Crude 10 (40 g) was dissolved into DMF (300 ml), and the mixture was cooled to $0{ }^{\circ} \mathrm{C}$. Imidazole (106 g, $1570 \mathrm{mmol})$ and tert-butyldimethylsilyl chloride $(100 \mathrm{~g}, 666 \mathrm{mmol})$ were added and the mixture was stirred at room temperature for $10 \mathrm{~h}$. $\mathrm{MeOH}(20 \mathrm{ml})$ was added and the mixture was stirred for $10 \mathrm{mim}$. The reaction mixture was diluted with $\mathrm{Et}_{2} \mathrm{O}$, washed with water and brine, and concentrated to give $\mathbf{1 1}(96 \mathrm{~g})$ as a pale yellow oil, which was used for the next reaction without purification. Pure 11, a colorless oil, obtained by silica gel column chromatography (hexane/AcOEt $=10 / 1-4 / 1$ ) showed the following spectral and analytical data: $[\alpha]_{\mathrm{D}}^{25}+53.7^{\circ}$ (c 1.20, $\mathrm{CHCl}_{3}$ ); FTIR (neat) 2939, 2858, 1462, 1251, $1089 \mathrm{~cm}^{-}$ ${ }^{1}$; ${ }^{1} \mathrm{H}$ NMR $\left(400 \mathrm{MHz}, \mathrm{CDCl}_{3}\right) \delta 5.76(\mathrm{dd}, J=2.0,10.4 \mathrm{~Hz}, 1 \mathrm{H}), 5.70(\mathrm{~d}, J=10.4 \mathrm{~Hz}, 1 \mathrm{H})$, $4.15(\mathrm{~m}, 3 \mathrm{H}), 3.89(\mathrm{dd}, J=2.0,11.2 \mathrm{~Hz}, 1 \mathrm{H}), 3.72(\mathrm{dd}, J=4.4,11.2 \mathrm{~Hz}, 1 \mathrm{H}), 3.29$ (t, $J=5.6$ $\mathrm{Hz}, 1 \mathrm{H}), 0.91(\mathrm{~s}, 9 \mathrm{H}), 0.90(\mathrm{~s}, 9 \mathrm{H}), 0.10(\mathrm{~s}, 3 \mathrm{H}), 0.10(\mathrm{~s}, 6 \mathrm{H}) ;{ }^{13} \mathrm{C} \mathrm{NMR}\left(100 \mathrm{MHz}, \mathrm{CDCl}_{3}\right) \delta$ $129.3,127.0,79.9,65.5,64.9,63.4,26.1,25.8,18.6,18.0,-4.1,-4.7,-5.0,-5.1$; HRMS (EI) calcd for $\mathrm{C}_{18} \mathrm{H}_{38} \mathrm{O}_{3} \mathrm{Si}_{2}\left(\mathrm{M}^{+}\right)$358.2360, found 358.2347.

Crude 11 (96 g) was dissolved into acetone $(522 \mathrm{ml})$ and water $(130 \mathrm{ml}) . \quad \mathrm{N}$ methylmolpholine oxide (NMO) $(61 \mathrm{~g}, 522 \mathrm{mmol})$ and $\mathrm{OsO}_{4}(1.5 \mathrm{M}$ in water, $8.7 \mathrm{ml}, 1.35$ mmol) were added, and the mixture was stirred for $15 \mathrm{~h}$. The reaction mixture was diluted with $\mathrm{Et}_{2} \mathrm{O}$, washed with $10 \% \mathrm{Na}_{2} \mathrm{~S}_{2} \mathrm{O}_{3}$, water, and brine, dried, concentrated, and filtrated thorough $\mathrm{SiO}_{2}(500 \mathrm{~g})$ with hexane/EtOAc (3:1) to give 12 (90 g) as a pale yellow solid, which was used for the next reaction without purification. Pure 12, a colorless solid, obtained by silica gel column chromatography (hexane/AcOEt $=4 / 1-2 / 1$ ) showed the following spectral and analytical data: $[\alpha]_{\mathrm{D}}^{24}+2.8^{\circ}\left(c 1.34, \mathrm{CHCl}_{3}\right)$; FTIR (neat) $3428,2942,2859,1463$, 
1251, $1103 \mathrm{~cm}^{-1} ;{ }^{1} \mathrm{H}$ NMR $\left(400 \mathrm{MHz}, \mathrm{CDCl}_{3}\right) \delta 4.00(\mathrm{dd}, J=2.2,12.4 \mathrm{~Hz}, 1 \mathrm{H}), 3.86(\mathrm{dd}, J=$ $2.0,11.2 \mathrm{~Hz}, 1 \mathrm{H}), 3.86(\mathrm{~m}, 1 \mathrm{H}), 3.75(\mathrm{dd}, J=4.9,11.2 \mathrm{~Hz}, 1 \mathrm{H}), 3.67(\mathrm{t}, J=9.0 \mathrm{~Hz}, 1 \mathrm{H}), 3.52$ $(\mathrm{dd}, J=1.0,12.4 \mathrm{~Hz}, 1 \mathrm{H}), 3.47(\mathrm{td}, J=9.0,3.5 \mathrm{~Hz}, 1 \mathrm{H}), 3.10(\mathrm{ddd}, J=2.0,4.9,9.0 \mathrm{~Hz}, 1 \mathrm{H})$, $2.38\left(\mathrm{~d}, J=9.0 \mathrm{~Hz}, 1 \mathrm{H}\right.$, exchangeable with $\left.\mathrm{D}_{2} \mathrm{O}\right), 2.19(\mathrm{~d}, J=7.1 \mathrm{~Hz}, 1 \mathrm{H}$, exchangeable with $\left.\mathrm{D}_{2} \mathrm{O}\right), 0.40(\mathrm{~s}, 18 \mathrm{H}), 0.16(\mathrm{~s}, 3 \mathrm{H}), 0.11(\mathrm{~s}, 3 \mathrm{H}), 0.08(\mathrm{~s}, 3 \mathrm{H}), 0.07(\mathrm{~s}, 3 \mathrm{H}) ;{ }^{13} \mathrm{C}$ NMR $(100 \mathrm{MHz}$, $\left.\mathrm{CDCl}_{3}\right) \delta 81.8,75.3,69.9,69.8,62.8,26.0,18.5,18.3,-3.9,-4.8,-5.0,-5.2$; HRMS (EI) calcd for $\mathrm{C}_{17} \mathrm{H}_{37} \mathrm{O}_{5} \mathrm{Si}_{2}\left[(\mathrm{M}-\mathrm{Me})^{+}\right]$377.2180, found 377.2173.

To an ice-cooled solution of crude $12(90 \mathrm{~g})$ in $\mathrm{CH}_{2} \mathrm{Cl}_{2}(500 \mathrm{ml})$ were added 2,2dimetoxypropane $(72 \mathrm{~g}, 690 \mathrm{mmol})$ and $p$-toluenesulfonic acid monohydrate $(2.2 \mathrm{~g}, 11.5$ mmol), and the mixture was stirred at $0{ }^{\circ} \mathrm{C}$ for $4 \mathrm{~h}$. After disappearance of $\mathbf{1 2}$ on TLC, MeOH $(11 \mathrm{~g}, 345 \mathrm{mmol})$ was added and stirring was continued at room temperature for $10 \mathrm{~h}$. The reaction mixture was diluted with $\mathrm{Et}_{2} \mathrm{O}$, washed with saturated $\mathrm{NaHCO}_{3}$ and brine, and concentrated. Recrystallization of the residue from hexane and column chromatography $\left(\mathrm{SiO}_{2}\right.$ $400 \mathrm{~g}$, hexane/AcOEt $=8 / 1)$ of the mother liqor gave $14\left(45.0 \mathrm{~g}, 54 \%\right.$ from 9): $[\alpha]_{\mathrm{D}}^{25}-40.9^{\circ}$ (c 1.92, $\mathrm{CHCl}_{3}$ ); FTIR (neat) 3460, 2943, 2860, 1458, 1378, 1315, 1249, $1134 \mathrm{~cm}^{-1}$; ${ }^{1} \mathrm{H}$ NMR $\left(400 \mathrm{MHz}, \mathrm{CDCl}_{3}\right) \delta 4.25(\mathrm{~d}, J=13.4 \mathrm{~Hz}, 1 \mathrm{H}), 4.17(\mathrm{dd}, J=6.2,2.0 \mathrm{~Hz}, 1 \mathrm{H}), 3.96(\mathrm{t}, J=6.2$ $\mathrm{Hz} 1 \mathrm{H}), 3.84(\mathrm{dd}, J=2.9,11.7 \mathrm{~Hz}, 1 \mathrm{H}), 3.73(\mathrm{dd}, J=2.2,13.4 \mathrm{~Hz}, 1 \mathrm{H}), 3.61(\mathrm{dd}, J=6.36$, $11.7 \mathrm{~Hz}, 1 \mathrm{H}), 3.59(\mathrm{dd}, J=6.2,9.5 \mathrm{~Hz}), 3.11$ (ddd, $J=3.0,6.4,9.3 \mathrm{~Hz}, 1 \mathrm{H}), 2.08($ brs, $1 \mathrm{H})$, $1.52(\mathrm{~s}, 3 \mathrm{H}), 1.35(\mathrm{~s}, 3 \mathrm{H}), 0.87(\mathrm{~s}, 9 \mathrm{H}), 0.15(\mathrm{~s}, 3 \mathrm{H}), 0.82(\mathrm{~s}, 3 \mathrm{H}) ;{ }^{13} \mathrm{C}$ NMR $(100 \mathrm{MHz}$, $\left.\mathrm{CDCl}_{3}\right) \delta 109.2,79.7,79.5,74.0,71.3,66.6,62.7,28.1,26.4,25.9,18.1,-4.0,-5.1$; HRMS (FAB) calcd for $\mathrm{C}_{15} \mathrm{H}_{3} \mathrm{O}_{5} \mathrm{Si}\left[(\mathrm{M}+\mathrm{H})^{+}\right] 319.1941$, found 319.1950 . 


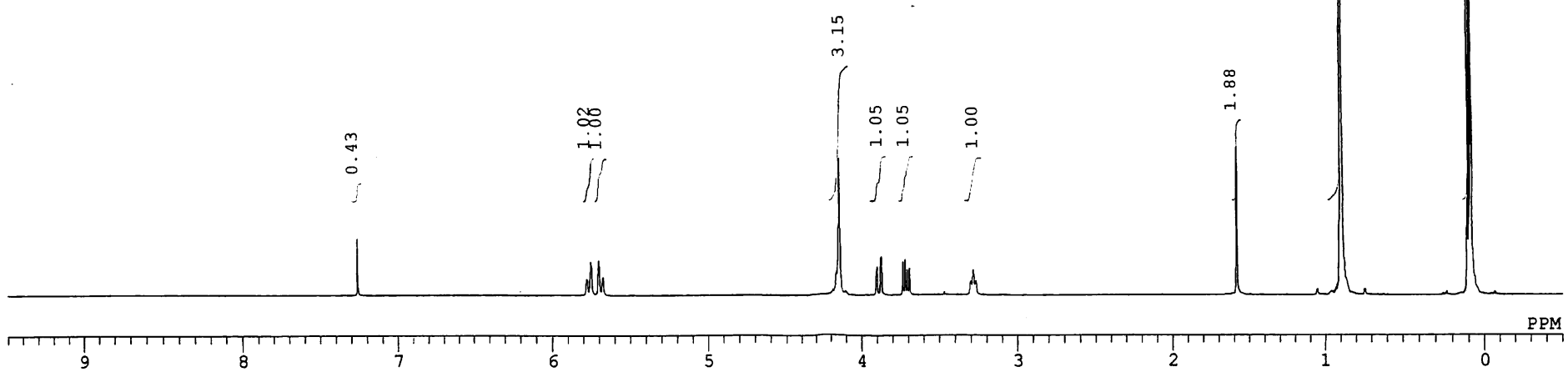

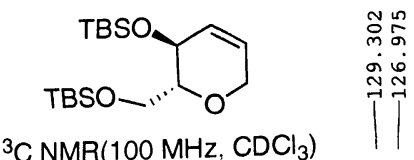

ํำ

ำ

${ }^{13} \mathrm{C} \mathrm{NMR}\left(100 \mathrm{MHz}, \mathrm{CDCl}_{3}\right)$

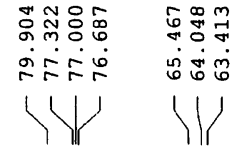

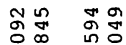

ํํำ

Y Y

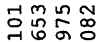

Ti个

If

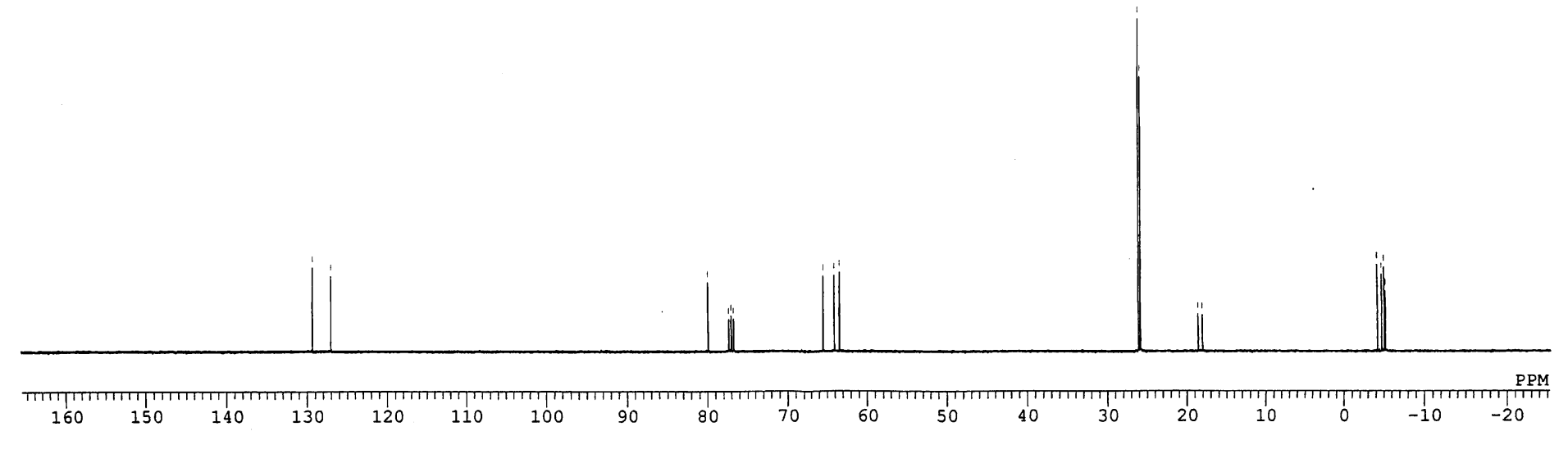




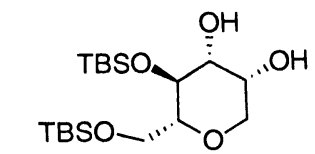

${ }^{1} \mathrm{H} \mathrm{NMR}\left(400 \mathrm{MHz}, \mathrm{CDCl}_{3}\right)$
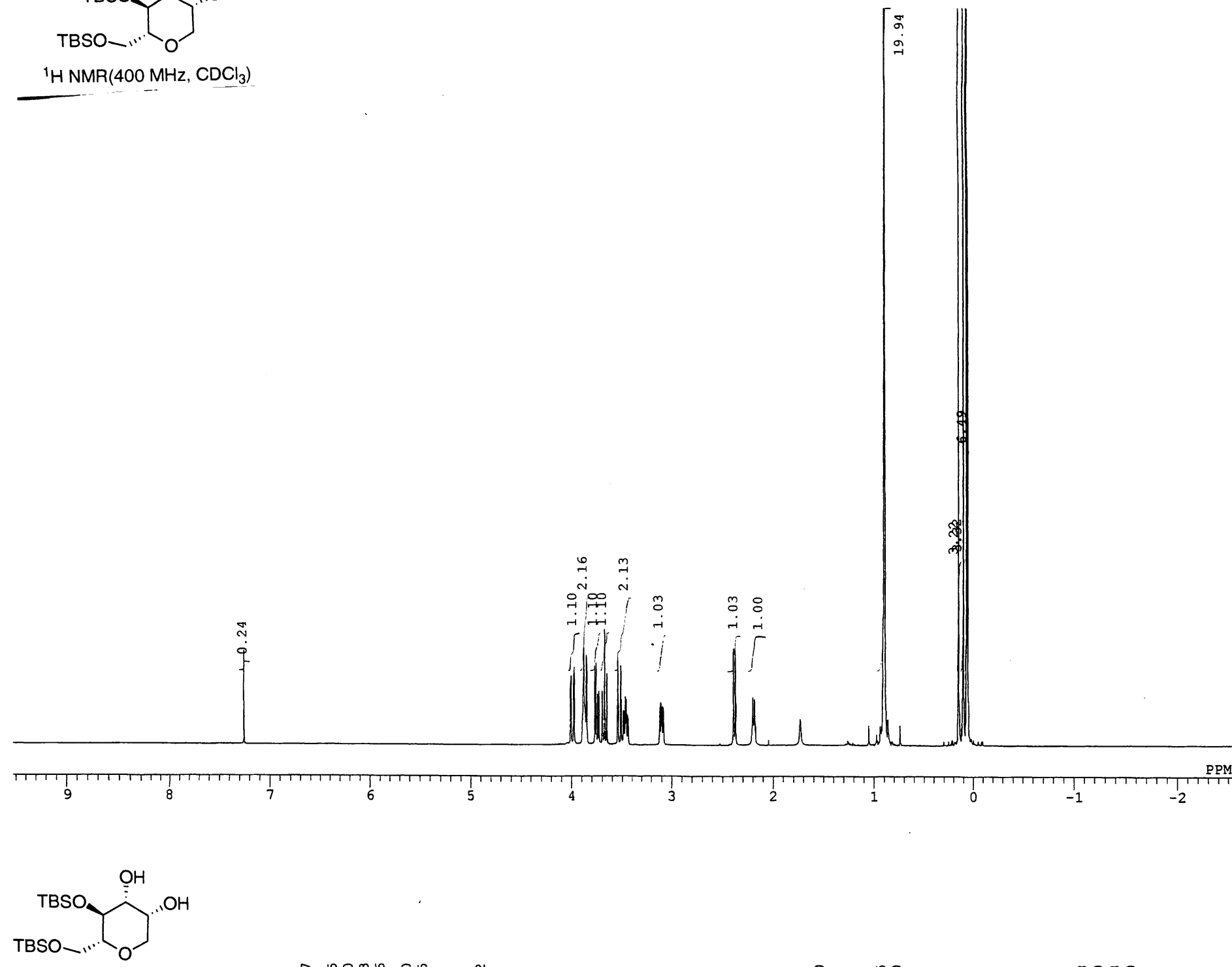

${ }^{13} \mathrm{C} \mathrm{NMR}\left(100 \mathrm{MHz}, \mathrm{CDCl}_{3}\right)$
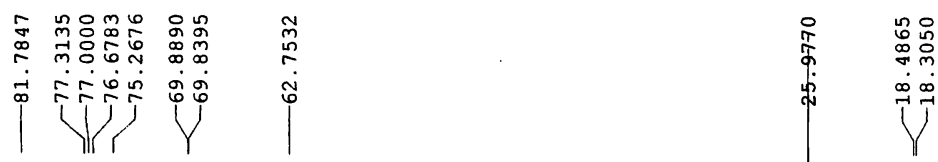

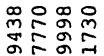

mंin

|

1)

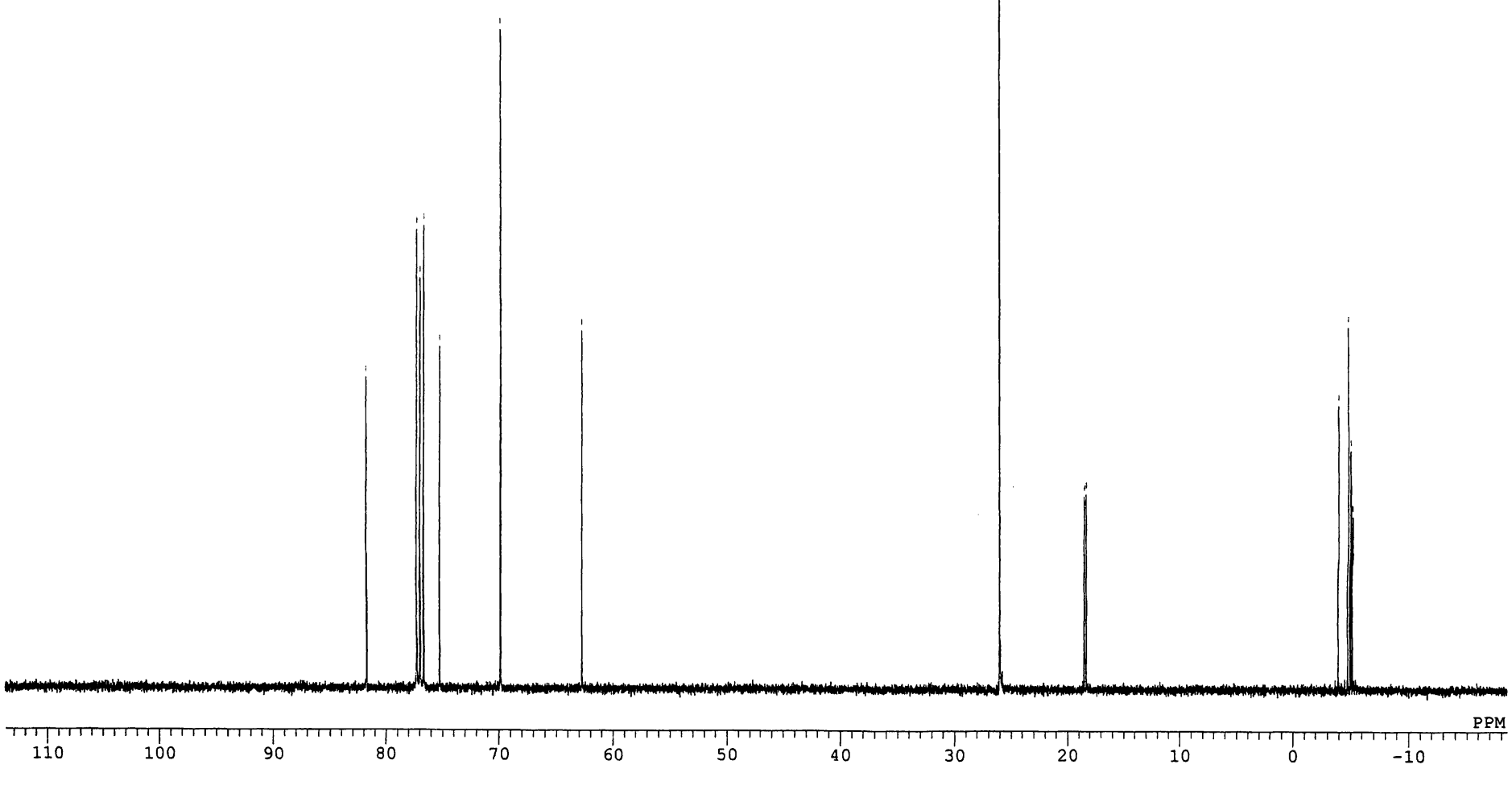




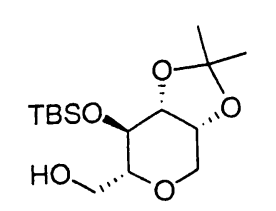

${ }^{1} \mathrm{H} \mathrm{NMR}\left(400 \mathrm{MHz}, \mathrm{CDCl}_{3}\right)$
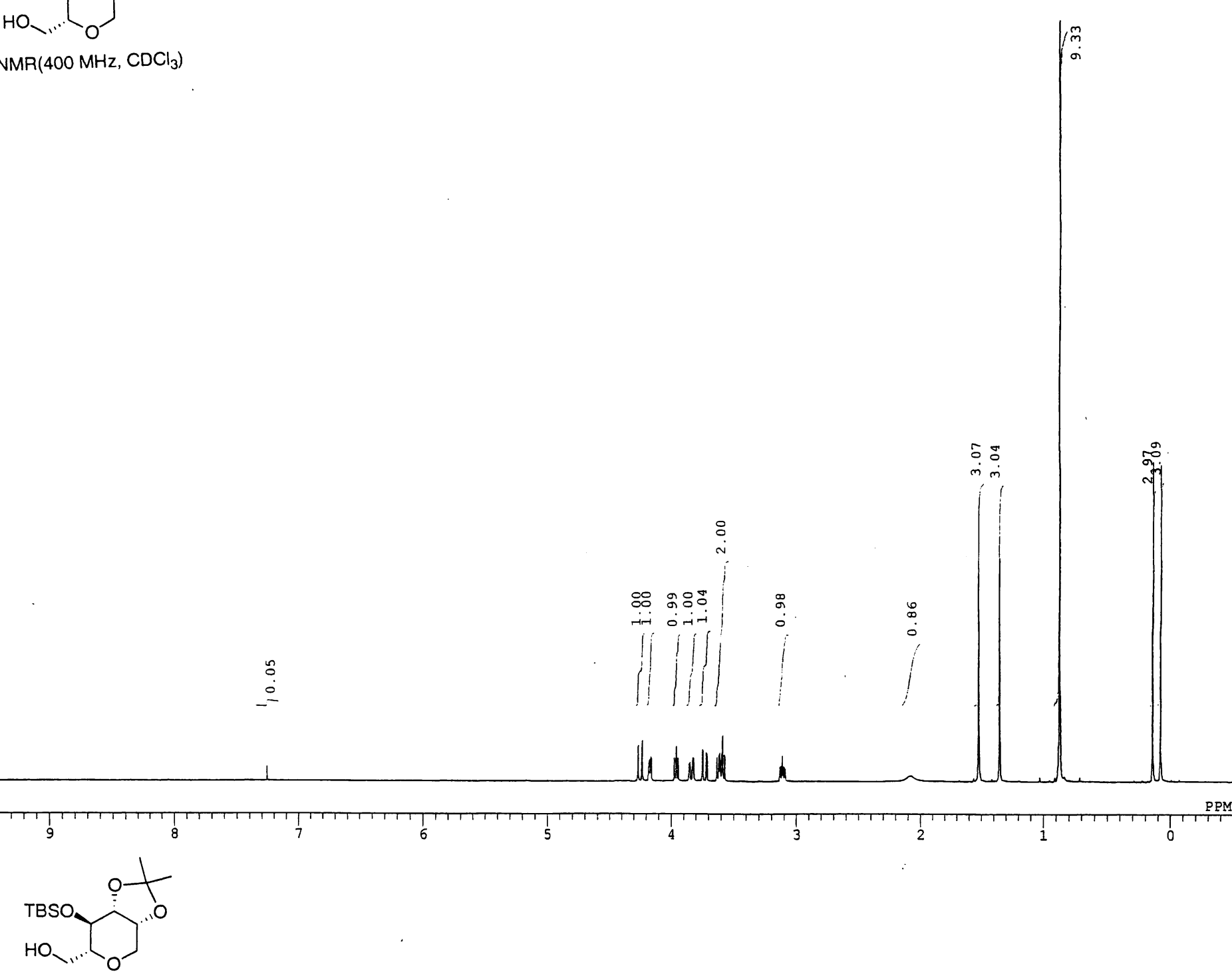

${ }^{13} \mathrm{C} \mathrm{NMR}\left(100 \mathrm{MHz}, \mathrm{CDCl}_{3}\right)$
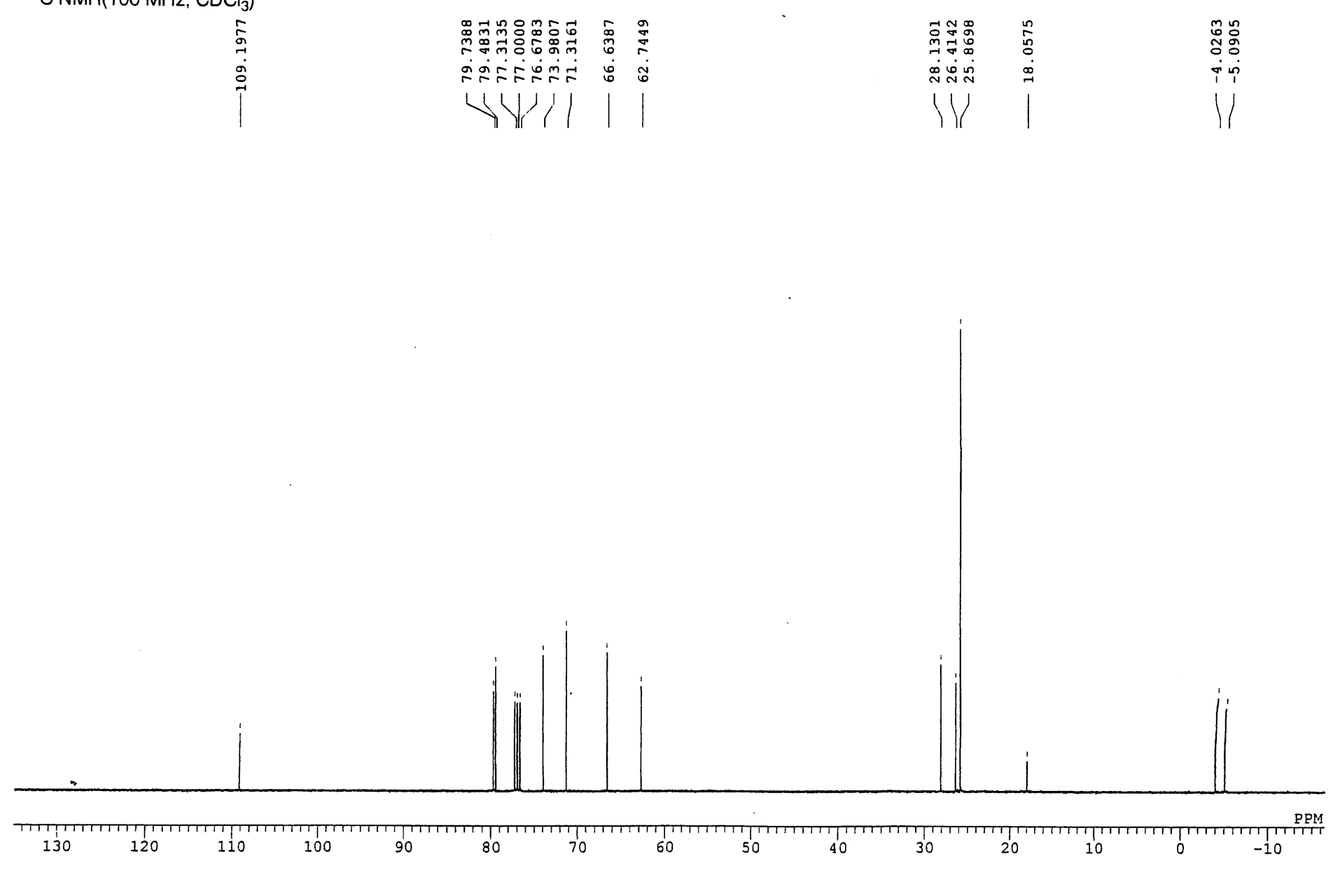
$\underbrace{1 .}_{15}$

${ }^{1} \mathrm{H} \mathrm{NMR}\left(400 \mathrm{MHz}, \mathrm{CDCl}_{3}\right)$

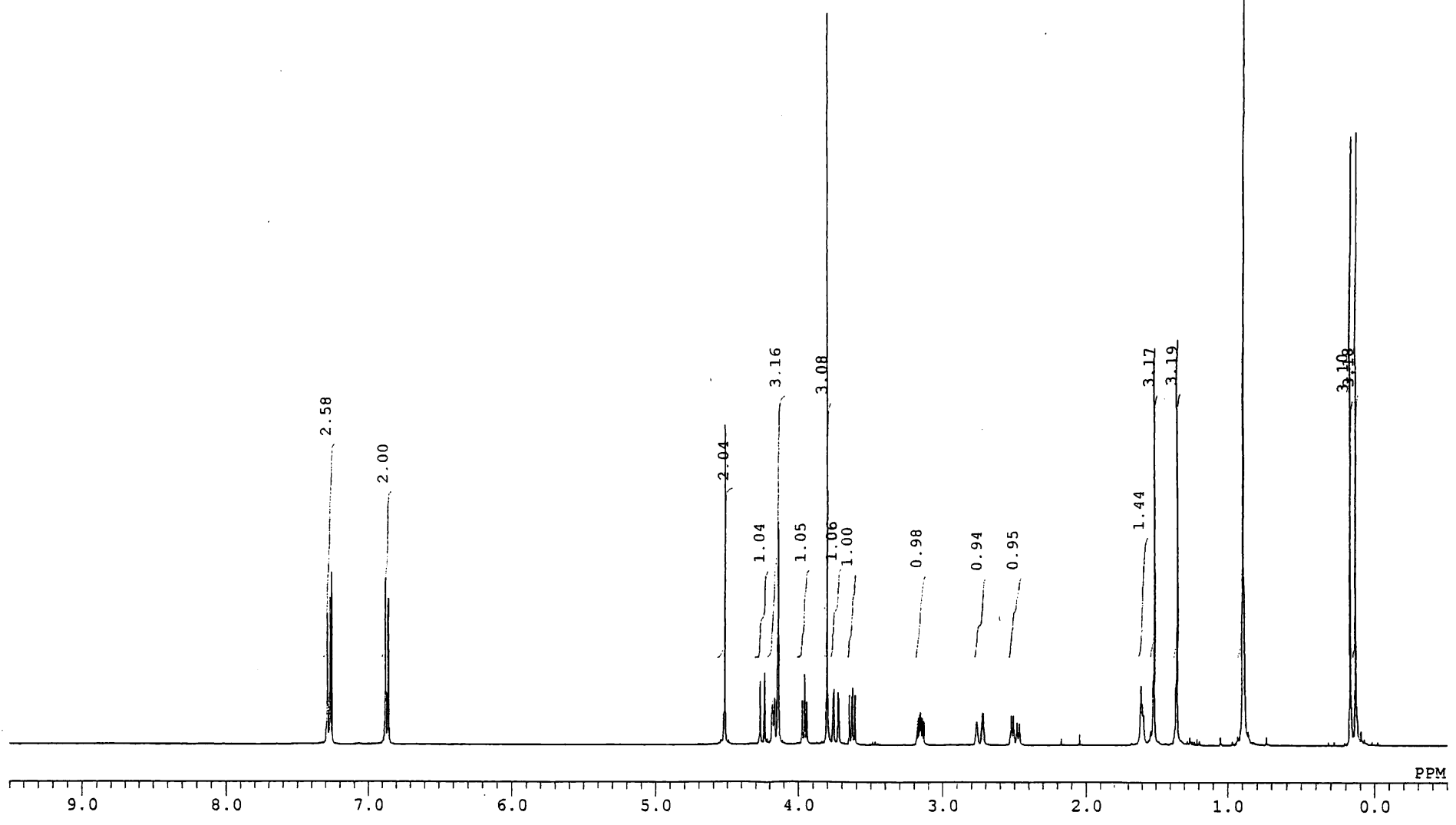

${ }^{13} \mathrm{C} \mathrm{NMR}\left(100 \mathrm{MHz}, \mathrm{CCl}_{3}\right)$

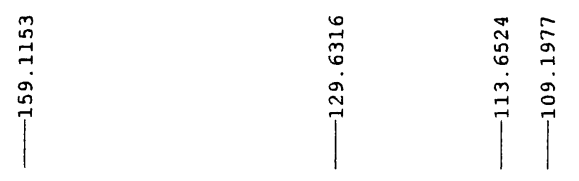

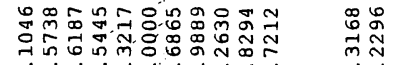

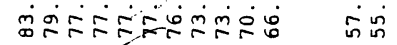

sif if !

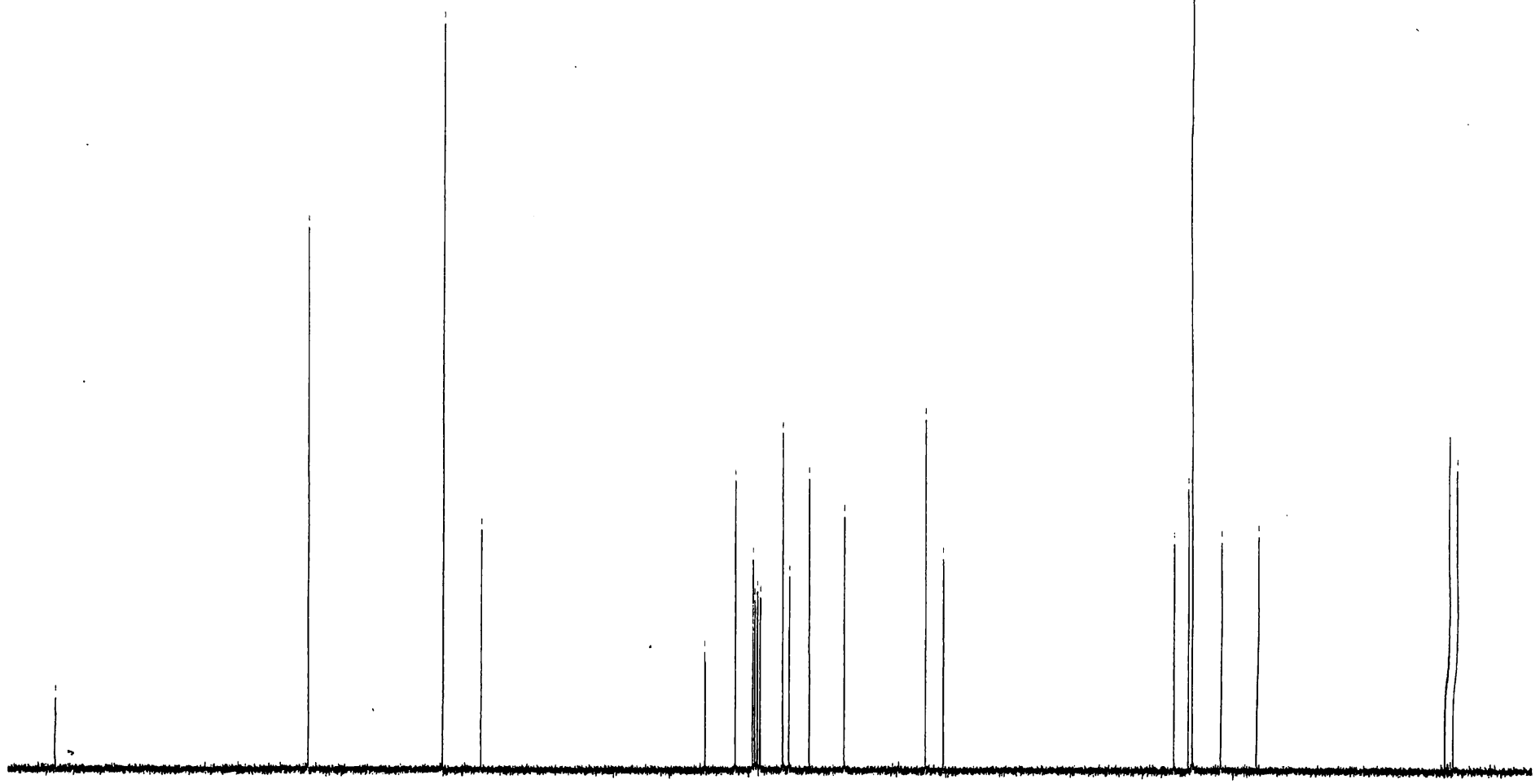

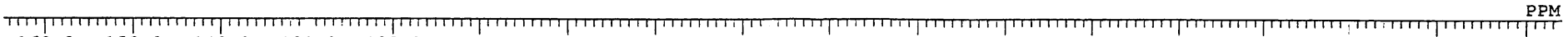

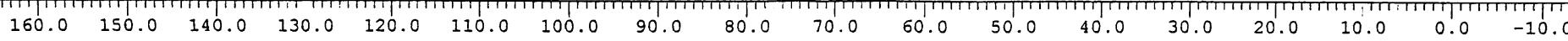
S7 
$\overbrace{16}^{\text {C.... }}$

'H NMR(400 MHz, $\mathrm{CDCl}_{3}$ )

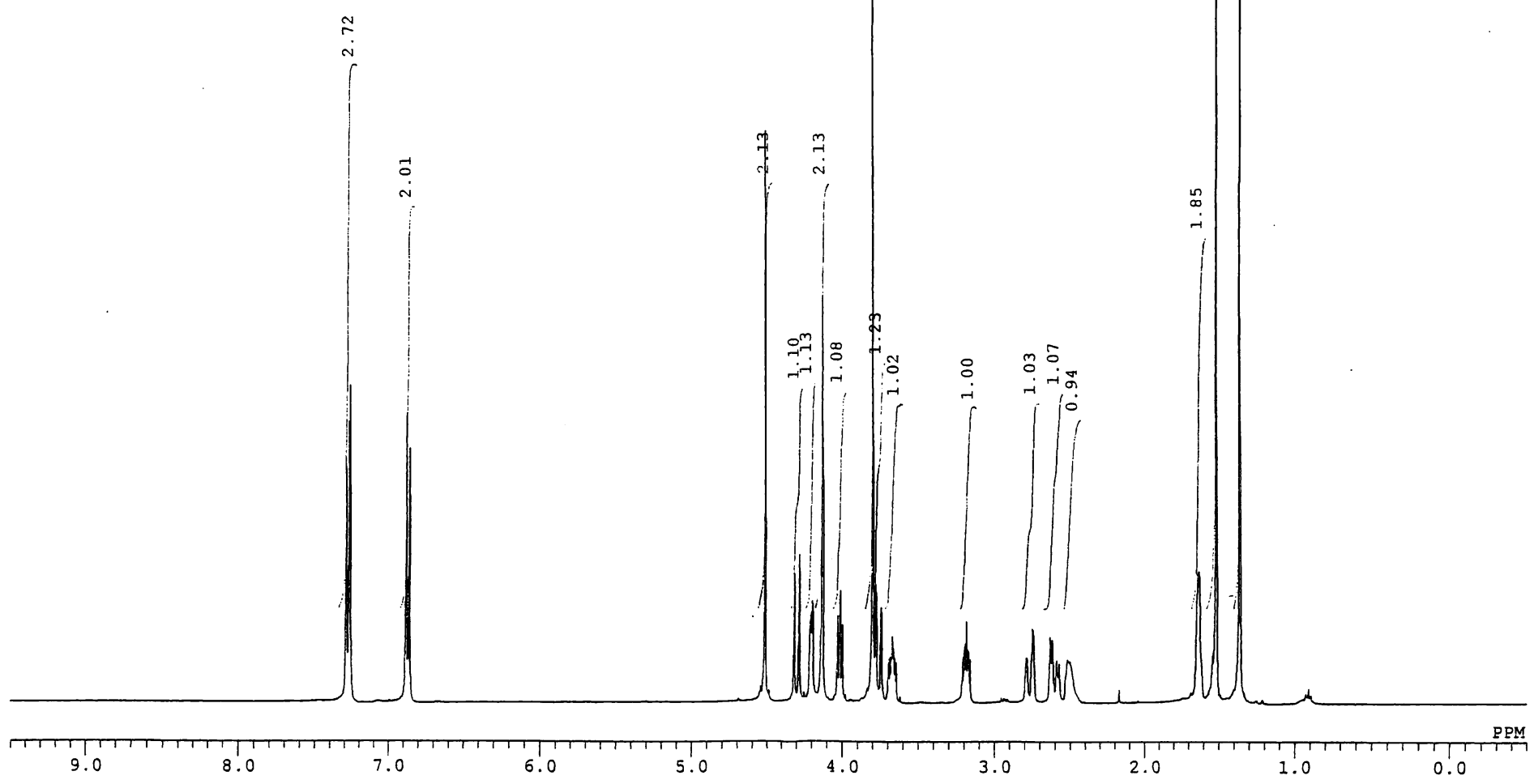<smiles>COCC#CC[C@H]1OC[C@@H]2OC(C)(C)O[C@H]2[C@H]1O</smiles>

${ }^{13} \mathrm{C} \mathrm{NMR}\left(100 \mathrm{MHz}, \mathrm{CDCl}_{3}\right)$

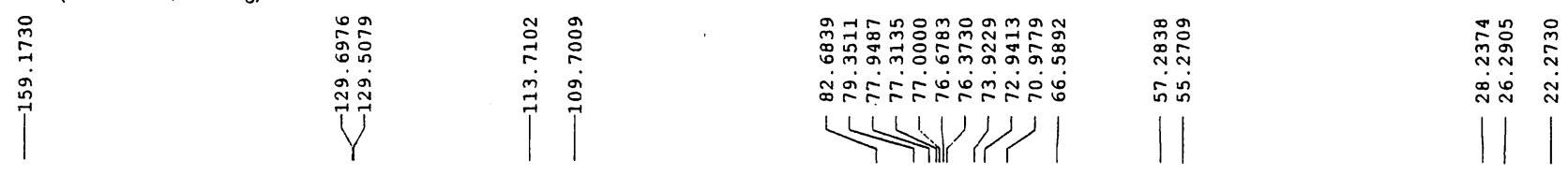

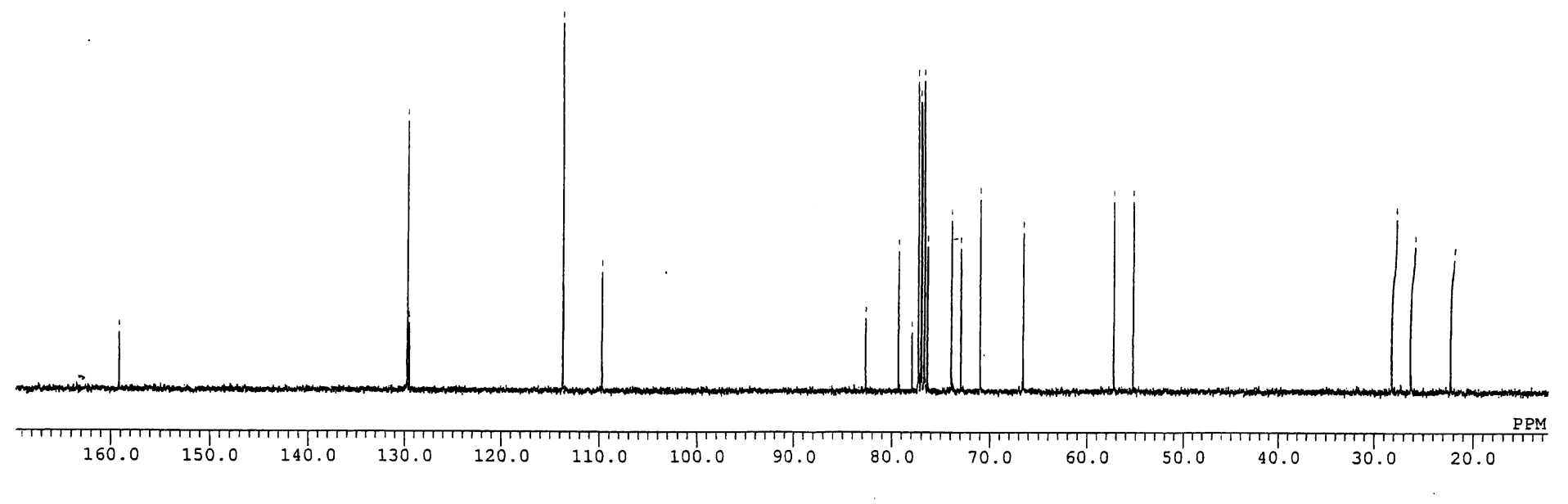




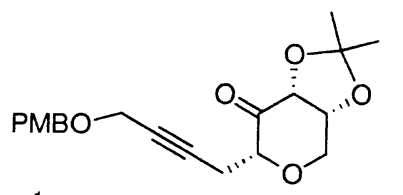

${ }^{1} \mathrm{H} \mathrm{NMR}\left(400 \mathrm{MHz}, \mathrm{CDCl}_{3}\right)$
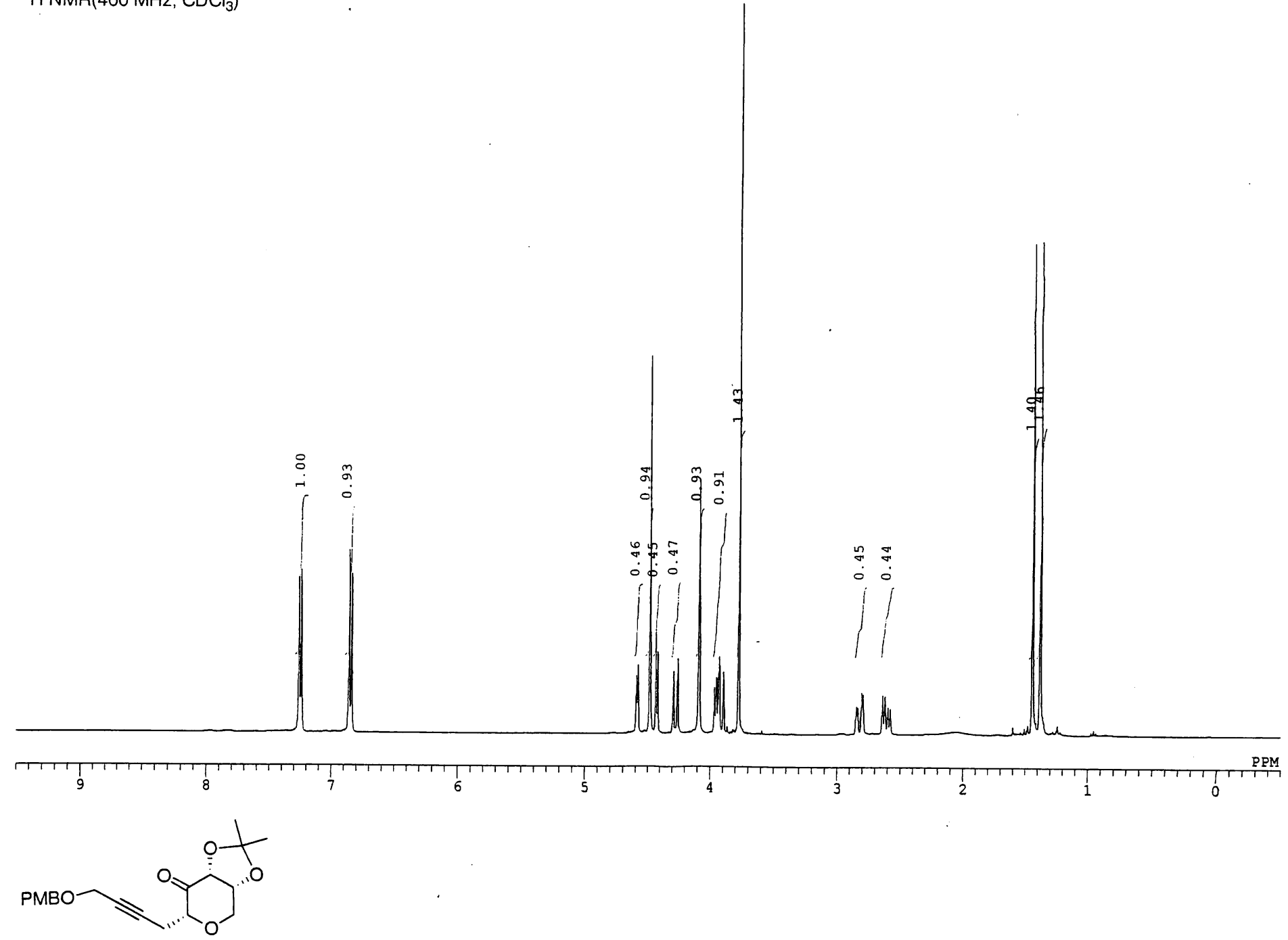

$\left.{ }^{13} \mathrm{C} \mathrm{NMR(100} \mathrm{MHz}, \mathrm{CDCl}_{3}\right)$
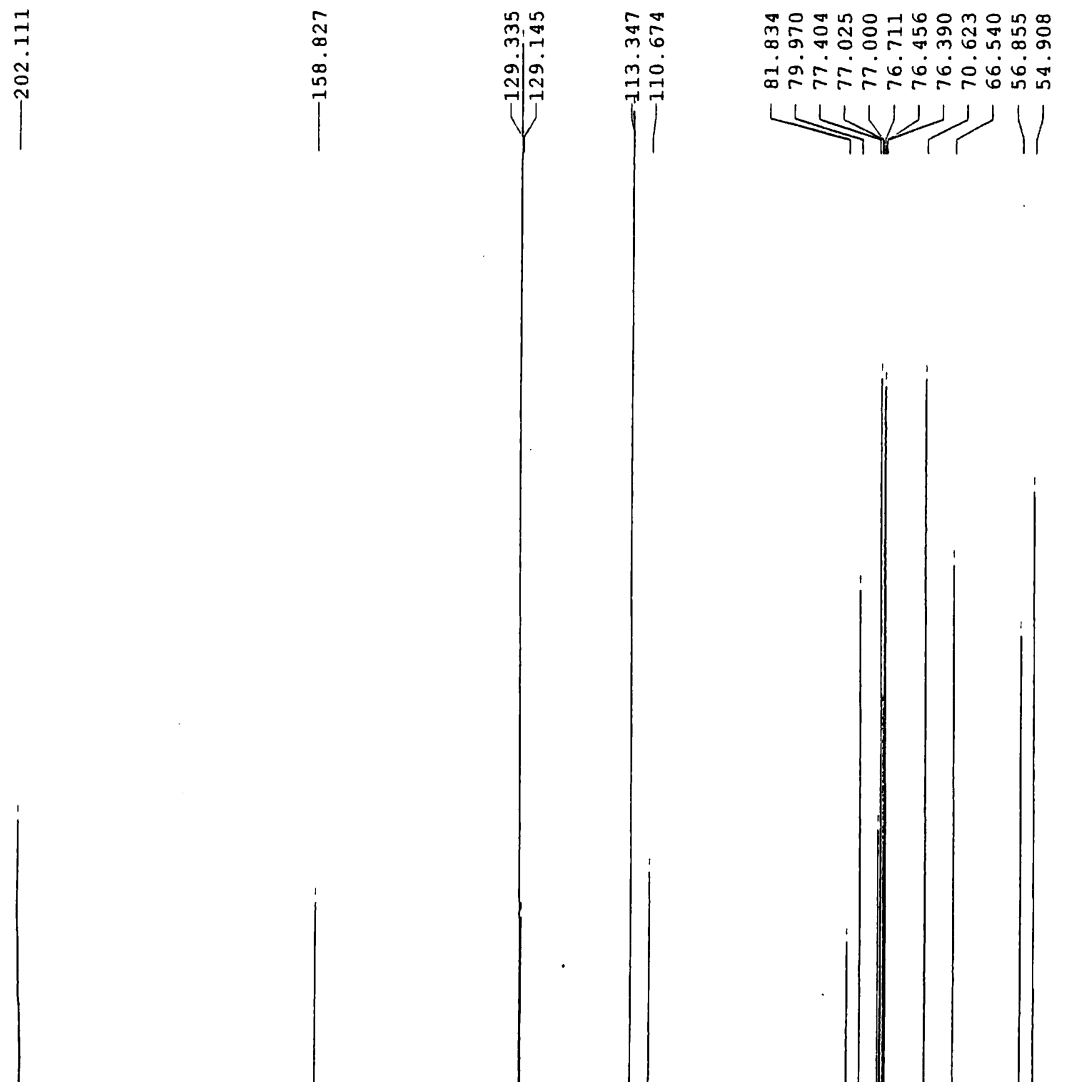

$\stackrel{\mathscr{D}}{\mathscr{m}} \mathbb{\Sigma}$

พับ ำ

|ri 
PMBO

${ }^{1} \mathrm{H} \mathrm{NMR}\left(400 \mathrm{MHz}, \mathrm{CDCl}_{3}\right)$
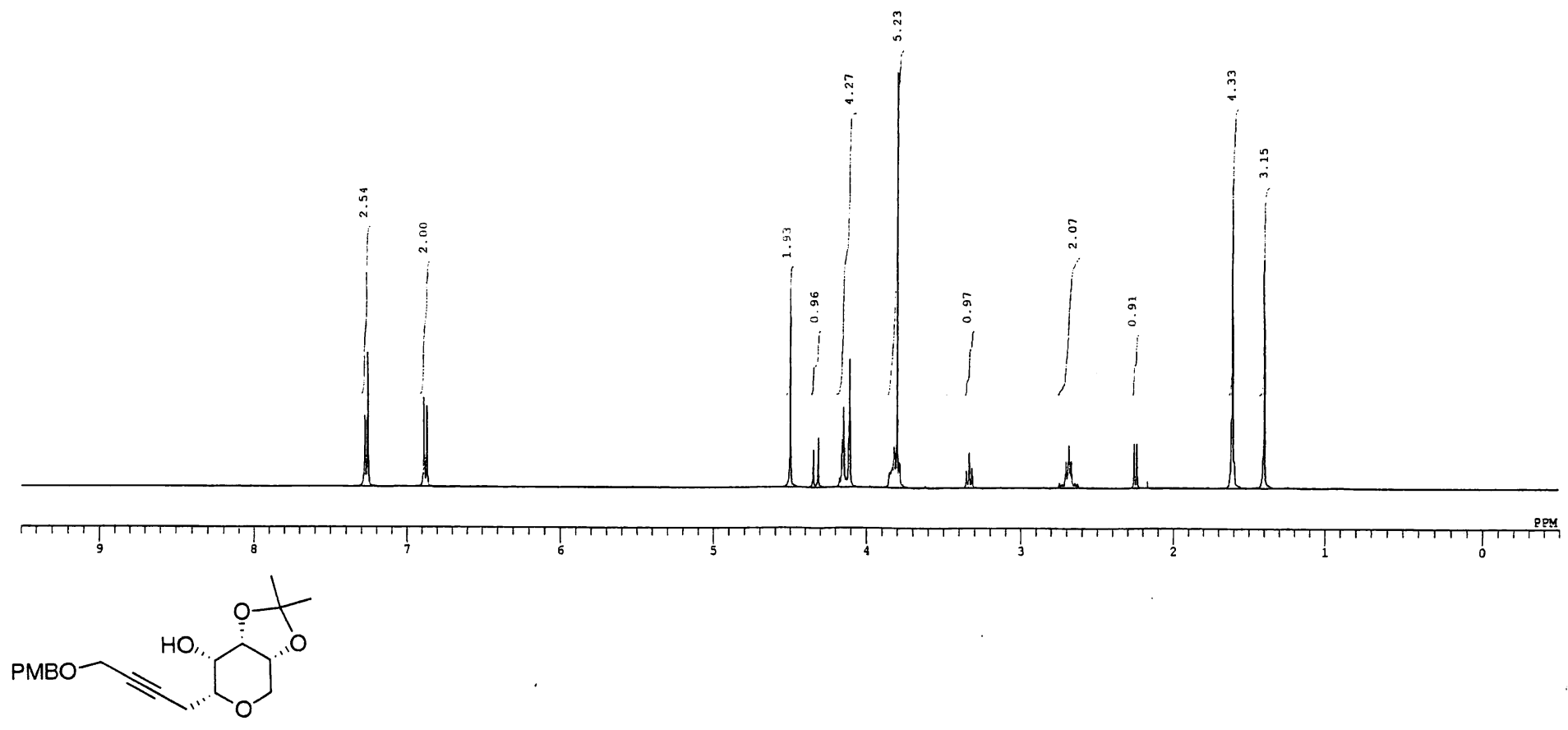

${ }^{13} \mathrm{C} \mathrm{NMR}\left(100 \mathrm{MHz}, \mathrm{CDCl}_{3}\right)$

$\prod_{\substack{1 \\ 0 \\ 0 \\ 0}}^{\substack{0 \\ 0}}$

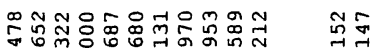

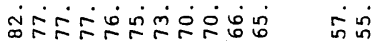

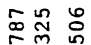

LWHIHU

มู่

Y)

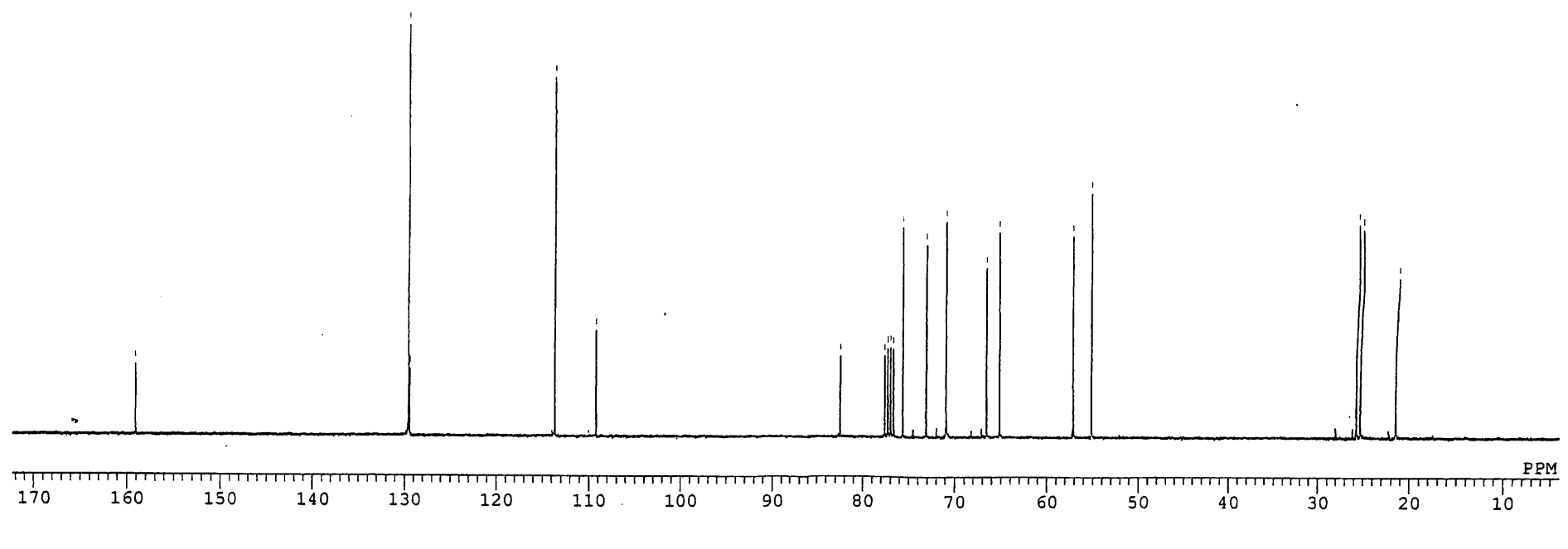


HESO,<smiles>CC1(C)OC2CCOCC2O1</smiles>

${ }^{1} \mathrm{H} \mathrm{NMR}\left(400 \mathrm{MHz}, \mathrm{CDCl}_{3}\right)$
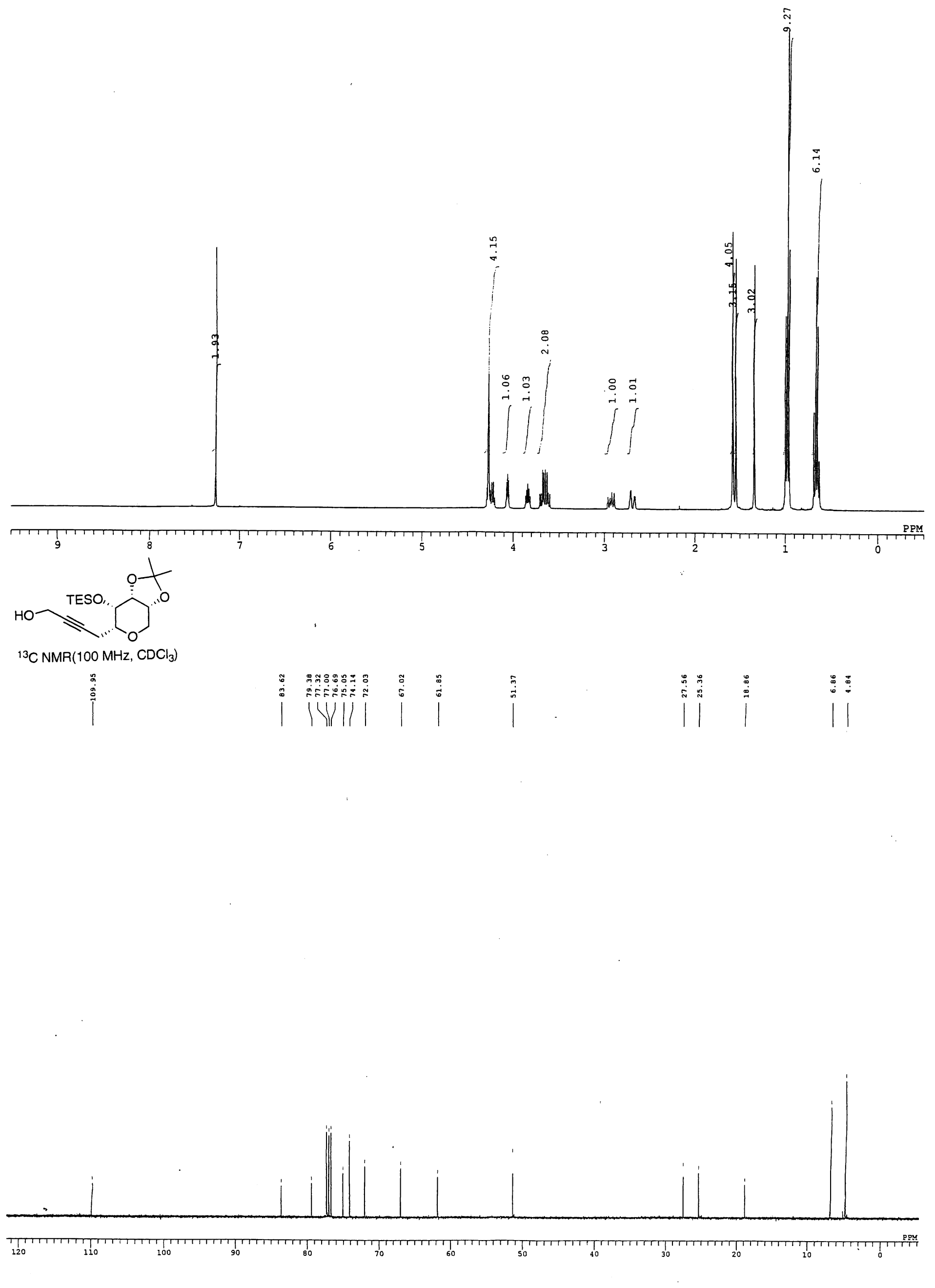

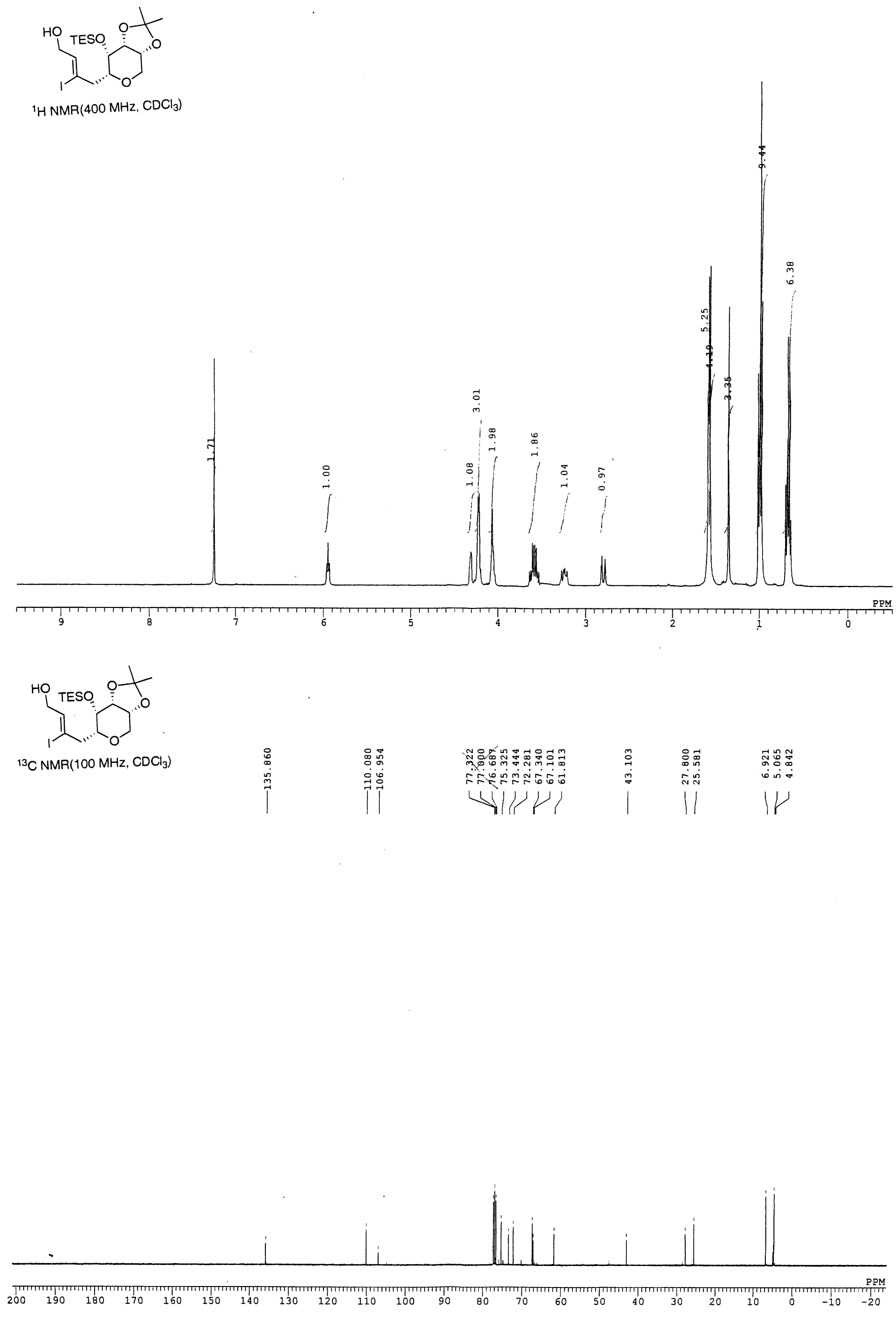


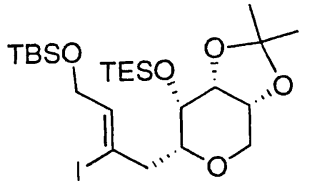

${ }^{1} \mathrm{H} \mathrm{NMR}\left(400 \mathrm{MHz}, \mathrm{CDCl}_{3}\right)$
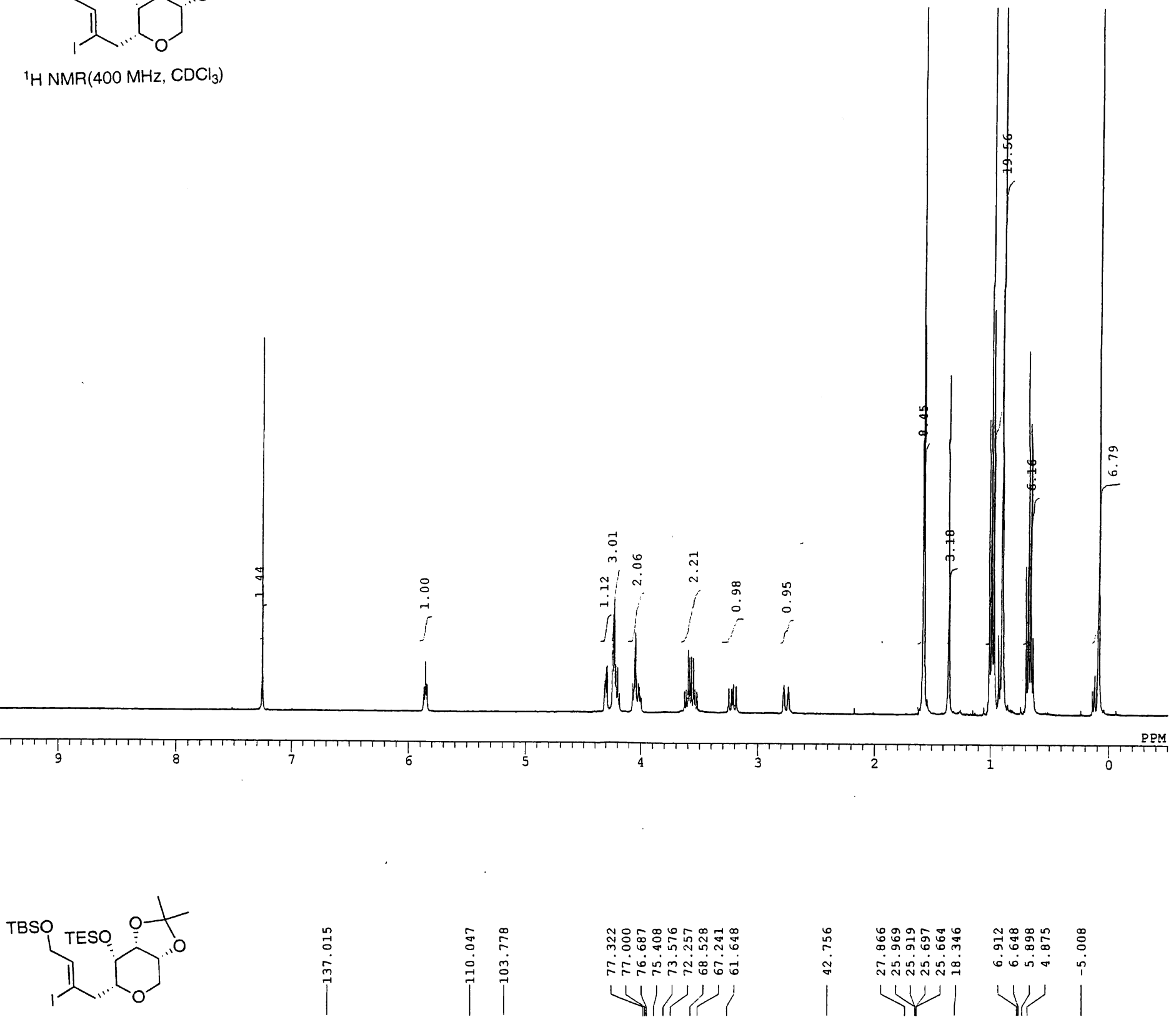

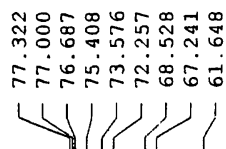

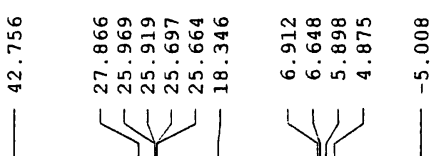

${ }^{13} \mathrm{C} \mathrm{NMR}\left(100 \mathrm{MHz}, \mathrm{CDCl}_{3}\right)$
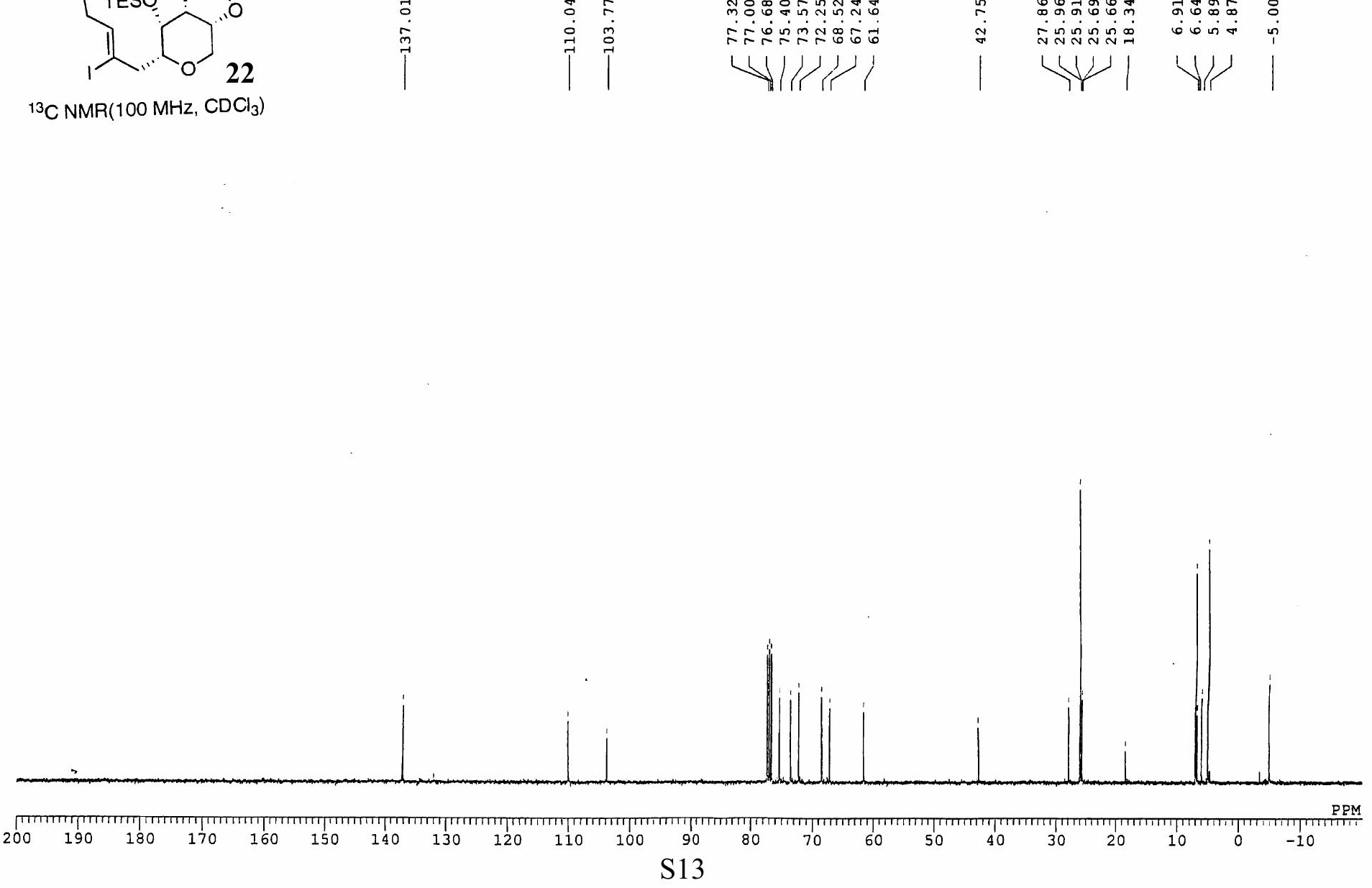

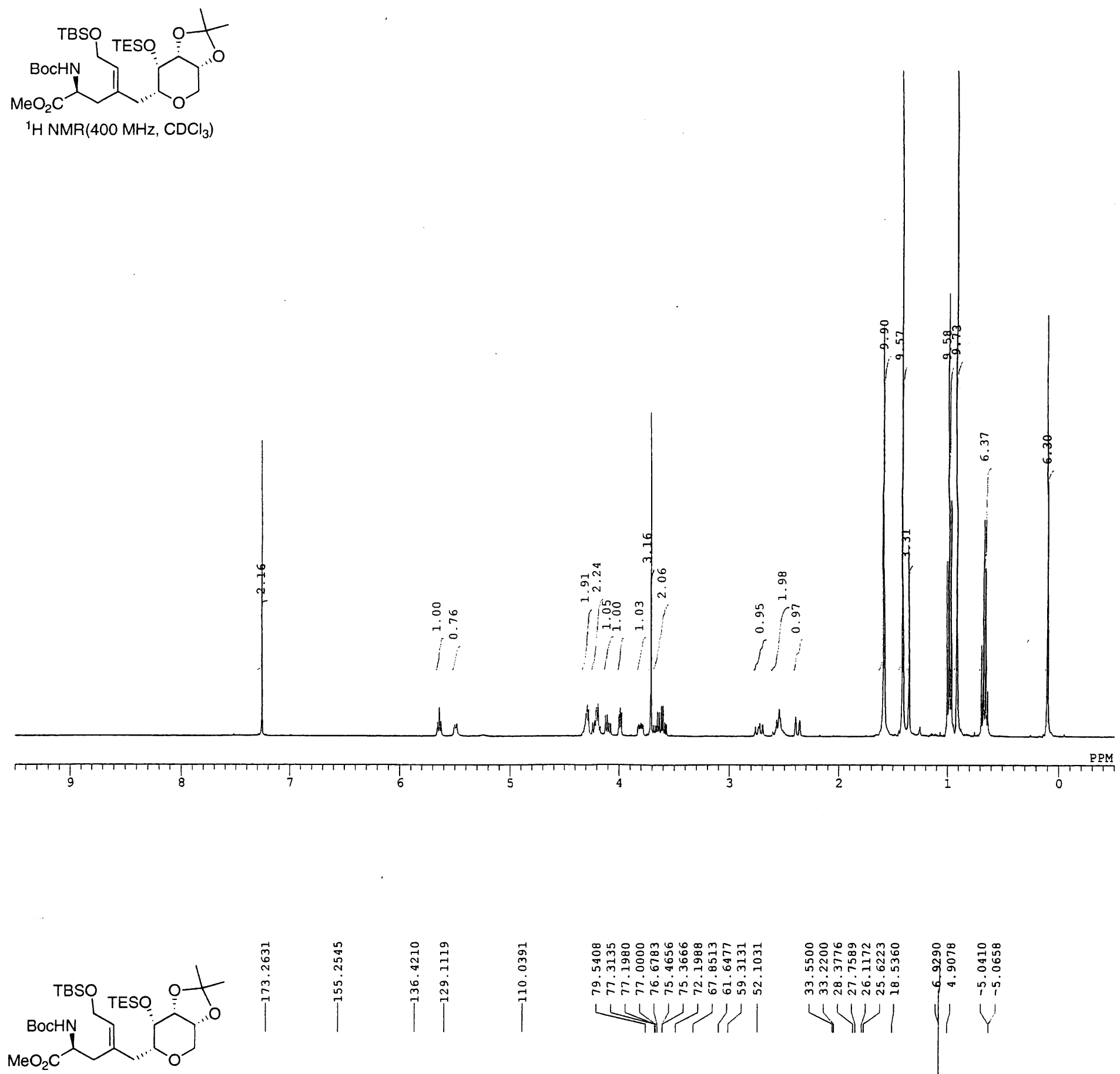

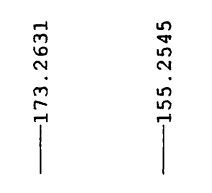

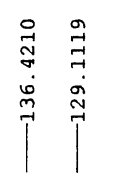

-1
ले
0
0
1

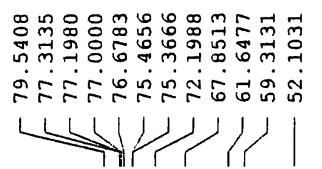

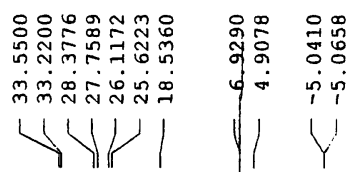

${ }^{13} \mathrm{C} \mathrm{NMR}\left(100 \mathrm{MHz}, \mathrm{CDCl}_{3}\right)$ 

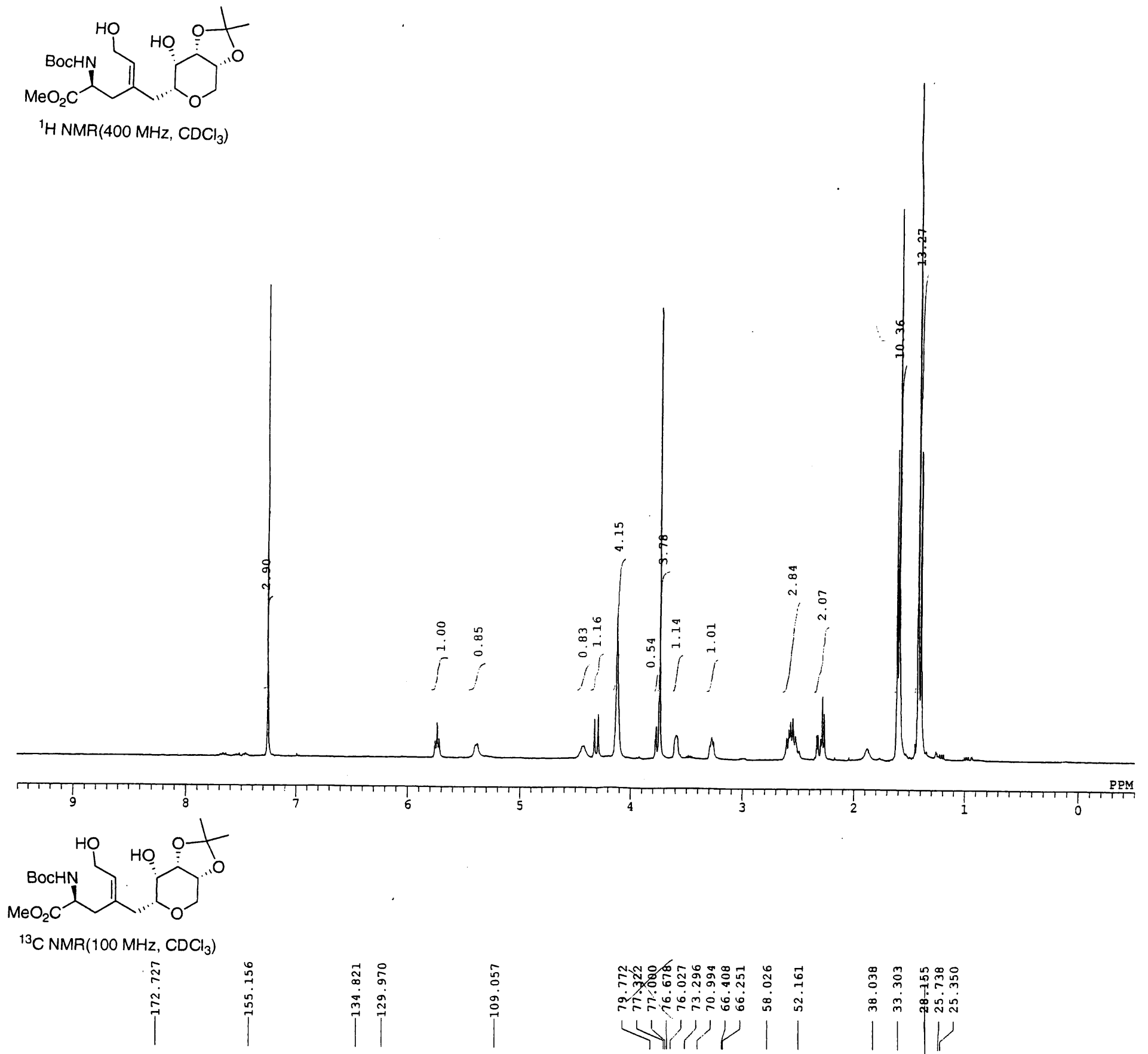

|
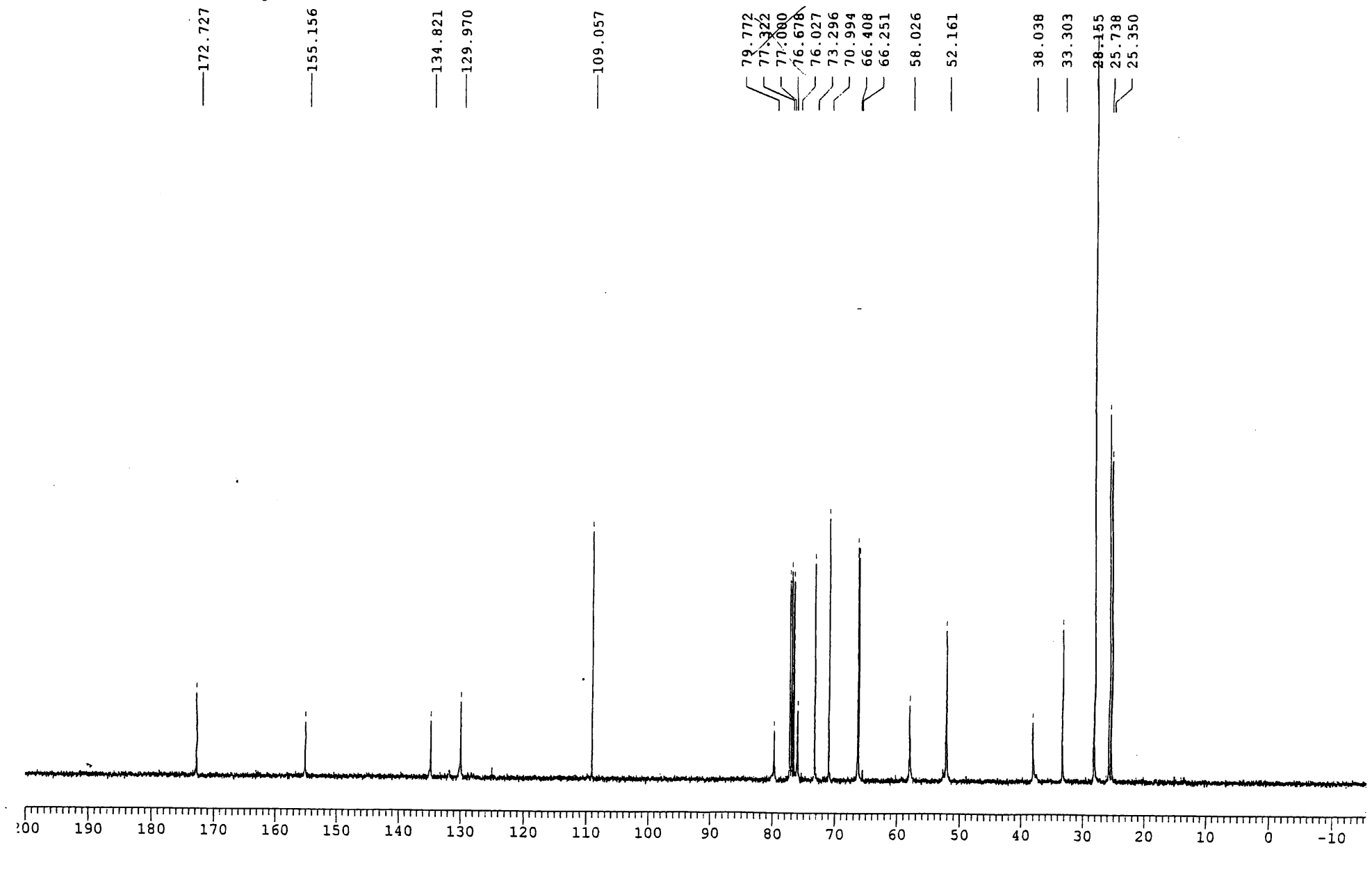


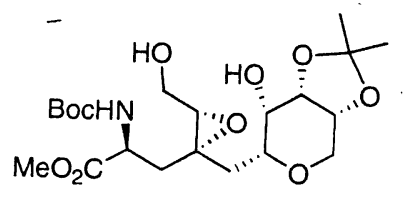

${ }^{1} \mathrm{H} \mathrm{NMR}\left(400 \mathrm{MHz}, \mathrm{CDCl}_{3}\right)$
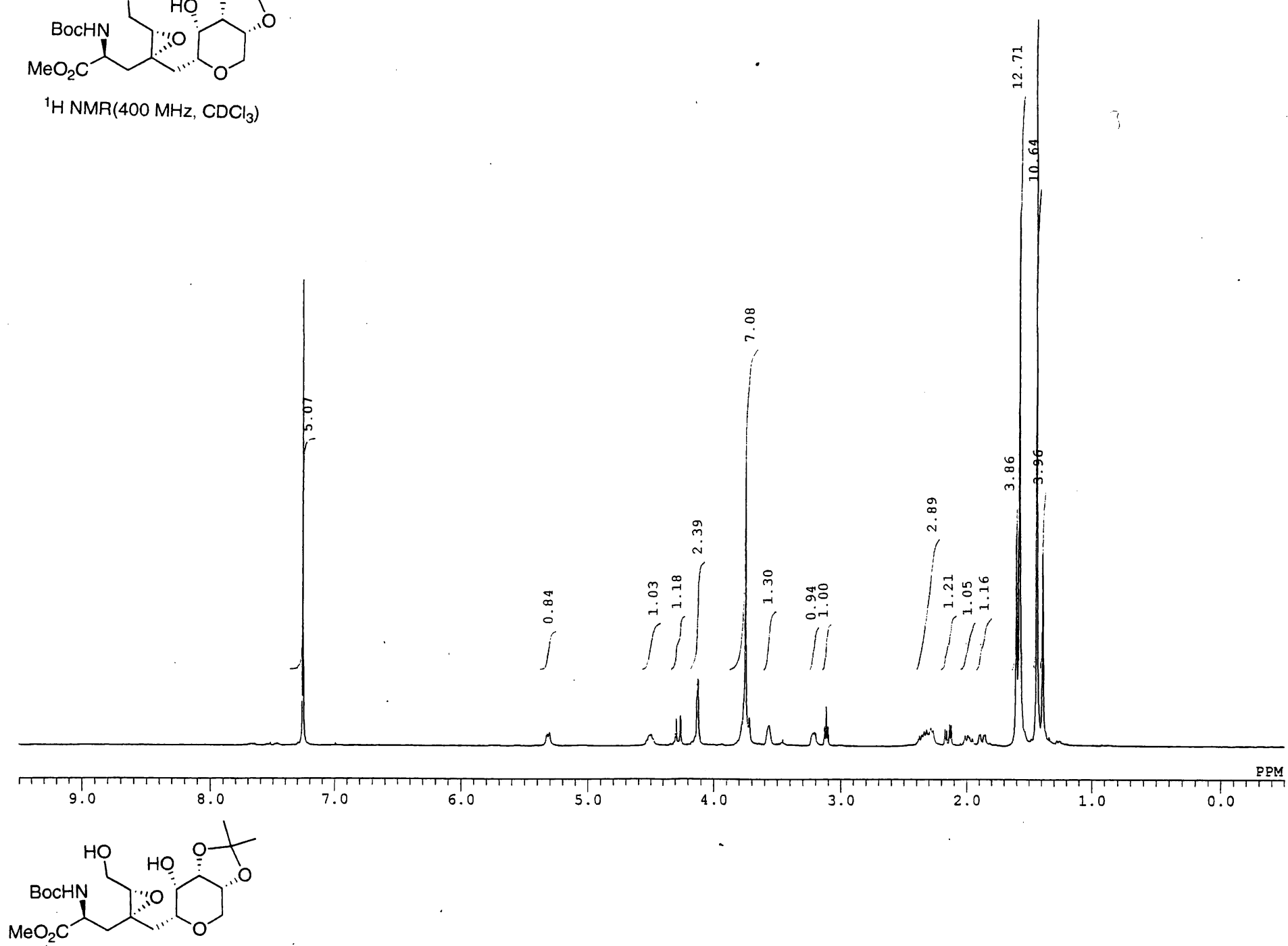

${ }^{13} \mathrm{C} \mathrm{NMR}\left(100 \mathrm{MHz}, \mathrm{CDCl}_{3}\right)$

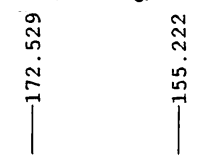

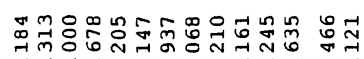

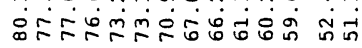

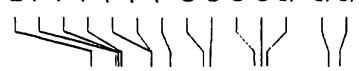

赵空

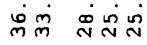

111

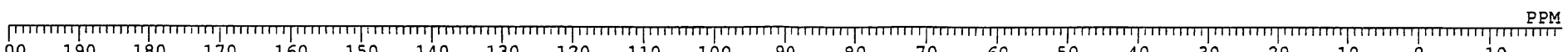




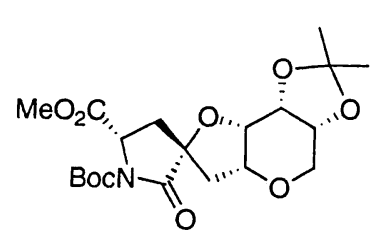

${ }^{1} \mathrm{HNMR}\left(500 \mathrm{MHz}, \mathrm{C}_{6} \mathrm{D}_{6}\right)$
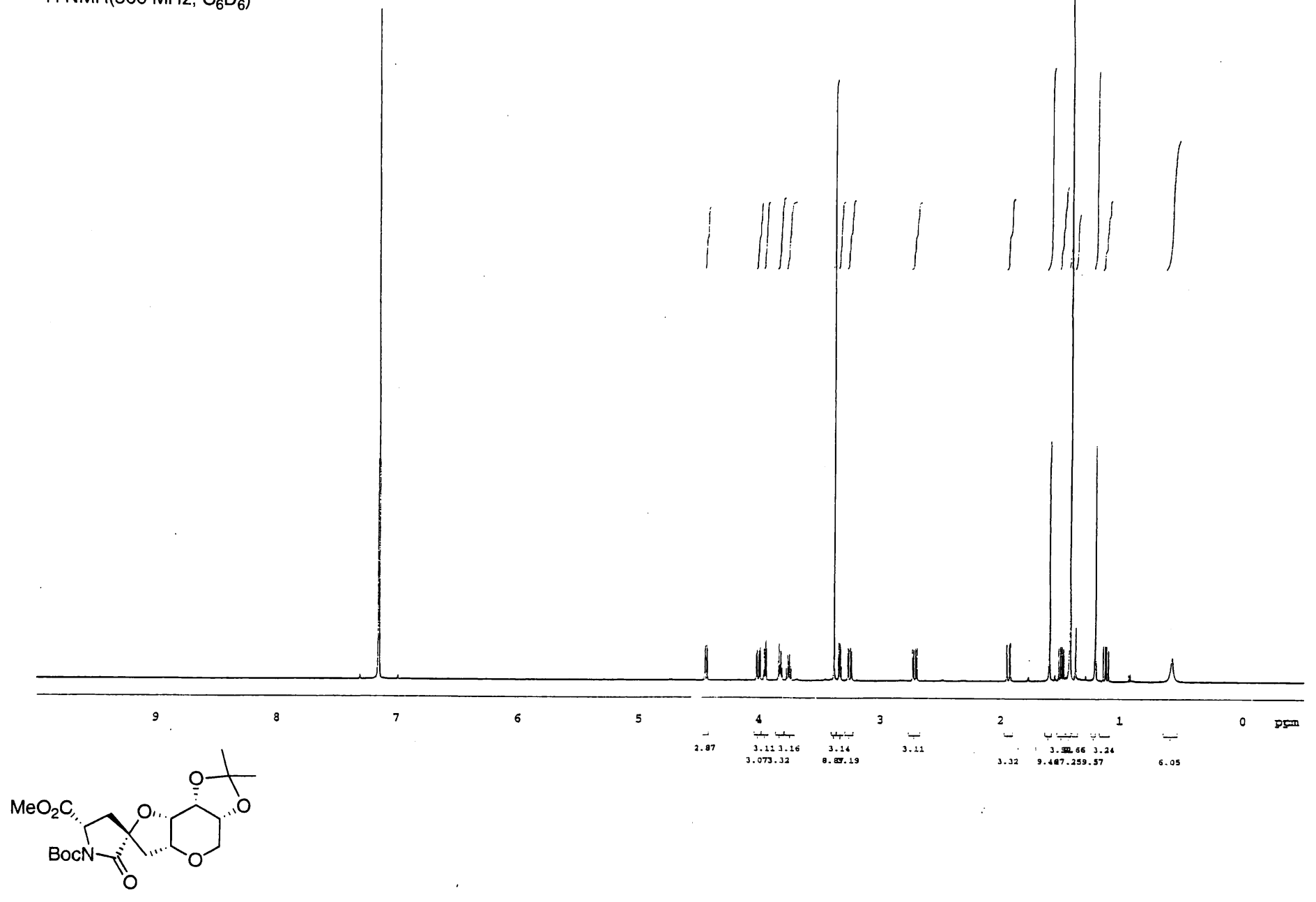

${ }^{13} \mathrm{C} \mathrm{NMR}\left(100 \mathrm{MHz}, \mathrm{CDCl}_{3}\right)$

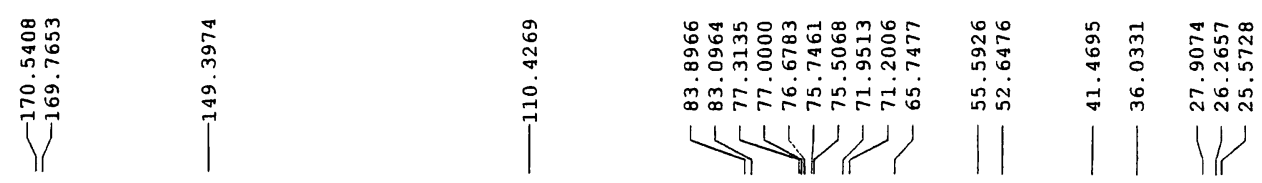

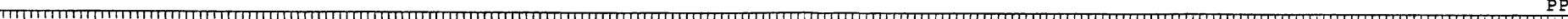




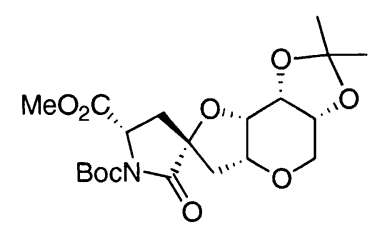

$\cos Y\left(500 \mathrm{MHz}, \mathrm{C}_{6} \mathrm{D}_{6}\right)$

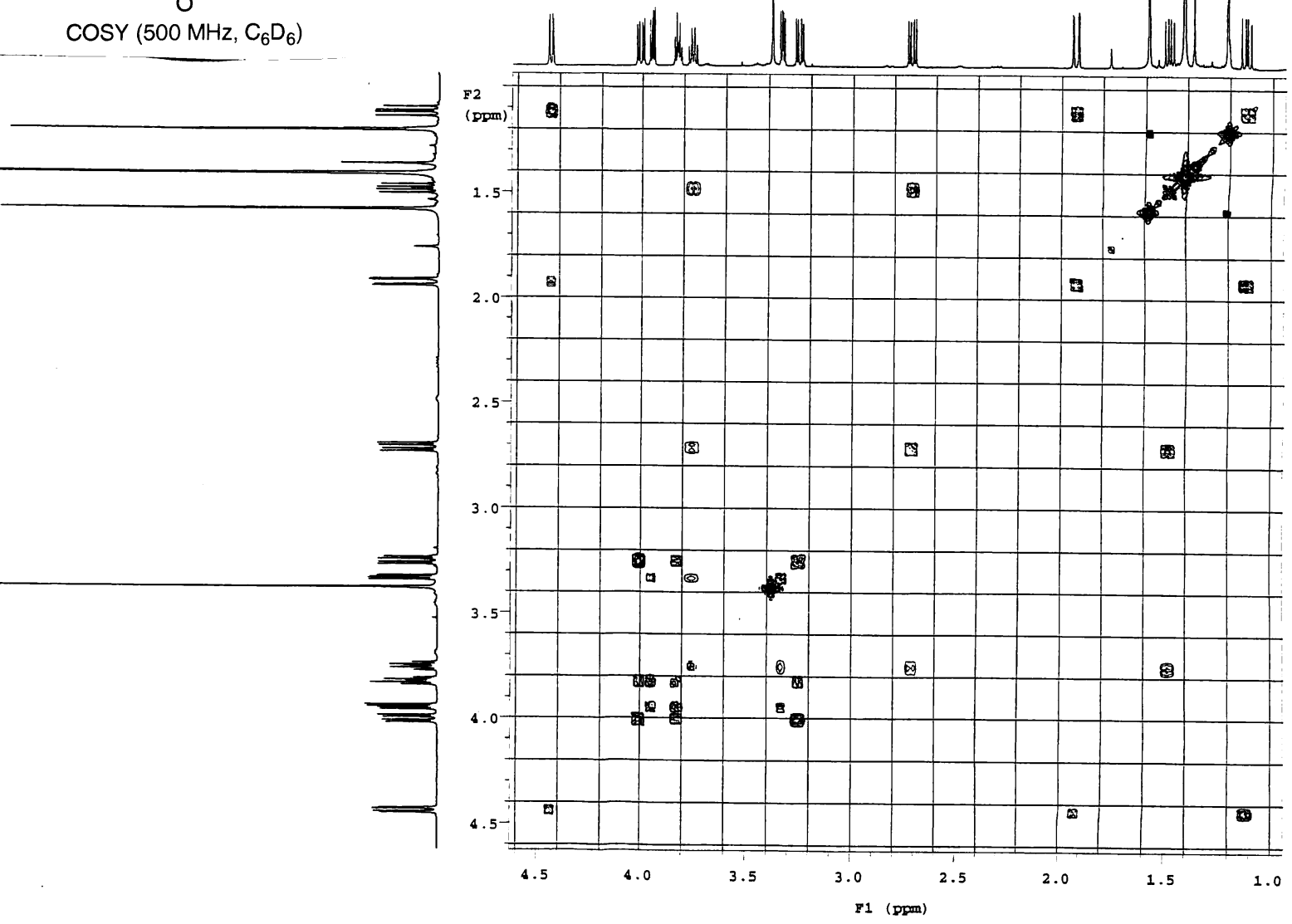

$\mathrm{MeO}_{2} \mathrm{C}$

NOESY $\left(500 \mathrm{MHz}, \mathrm{C}_{6} \mathrm{D}_{6}\right)$
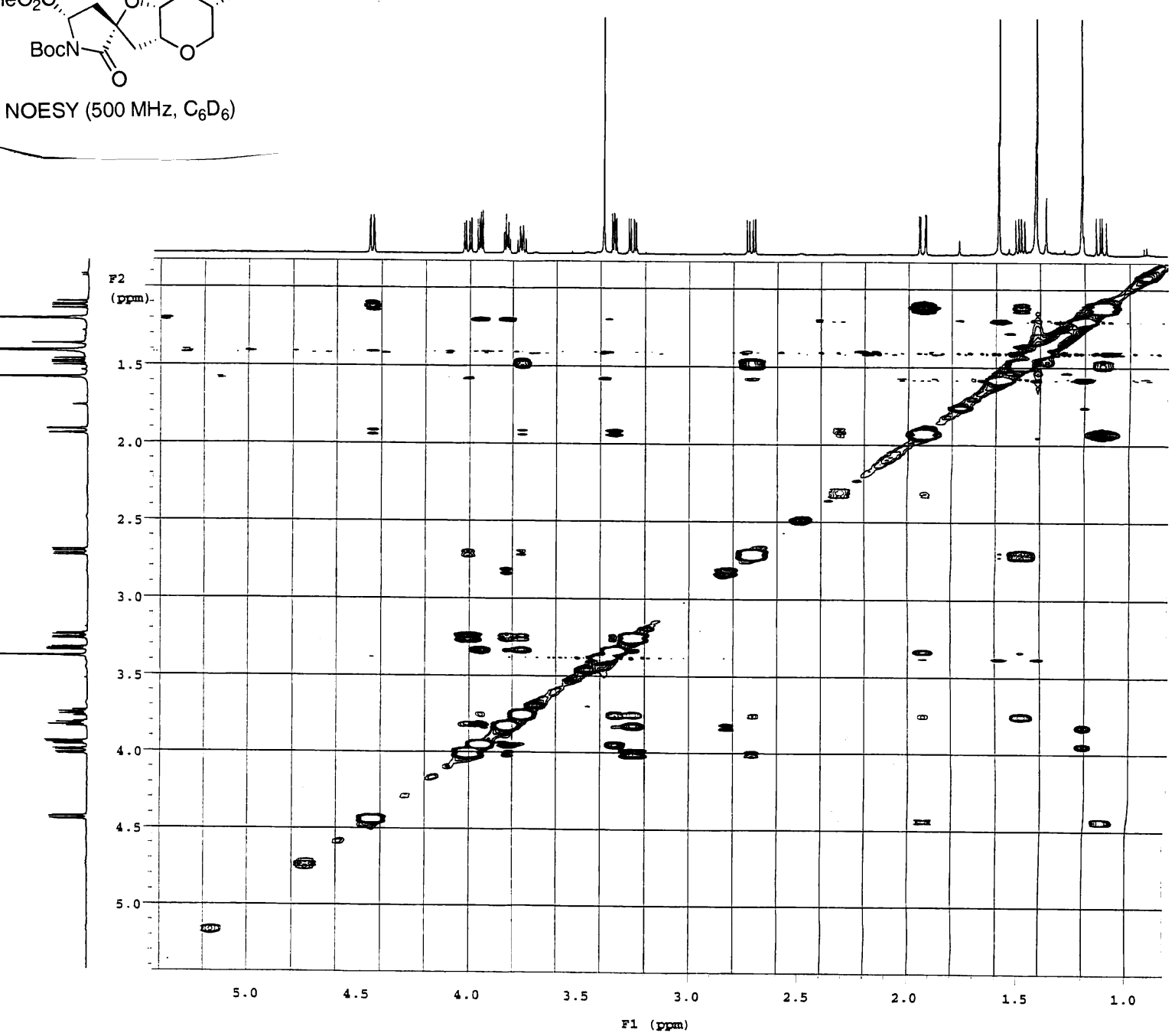


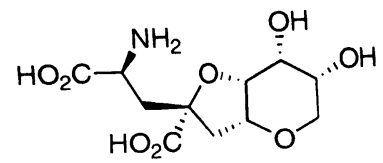

${ }^{1} \mathrm{H} \mathrm{NMR}\left(500 \mathrm{MHz}, \mathrm{D}_{2} \mathrm{O}\right)$
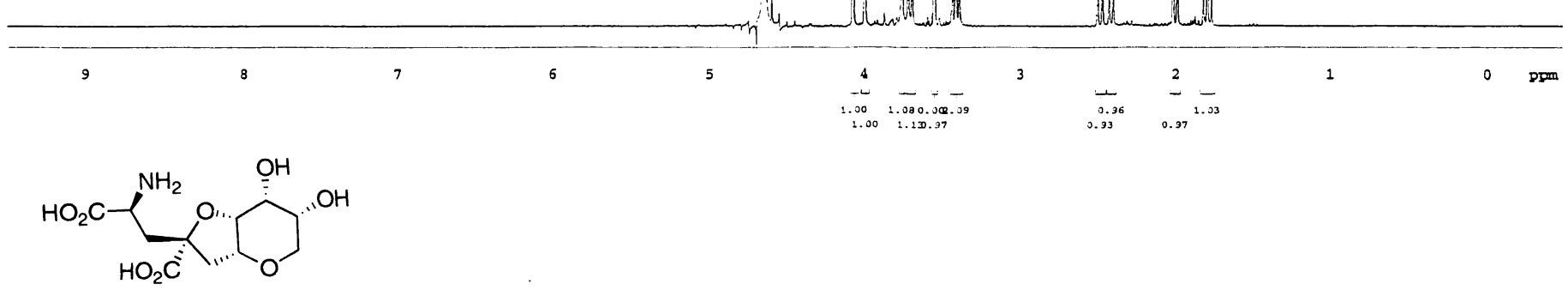

${ }^{13} \mathrm{C} \mathrm{NMR}\left(100 \mathrm{MHz}, \mathrm{D}_{2} \mathrm{O}+\mathrm{CD}_{3} \mathrm{OD}\right)$
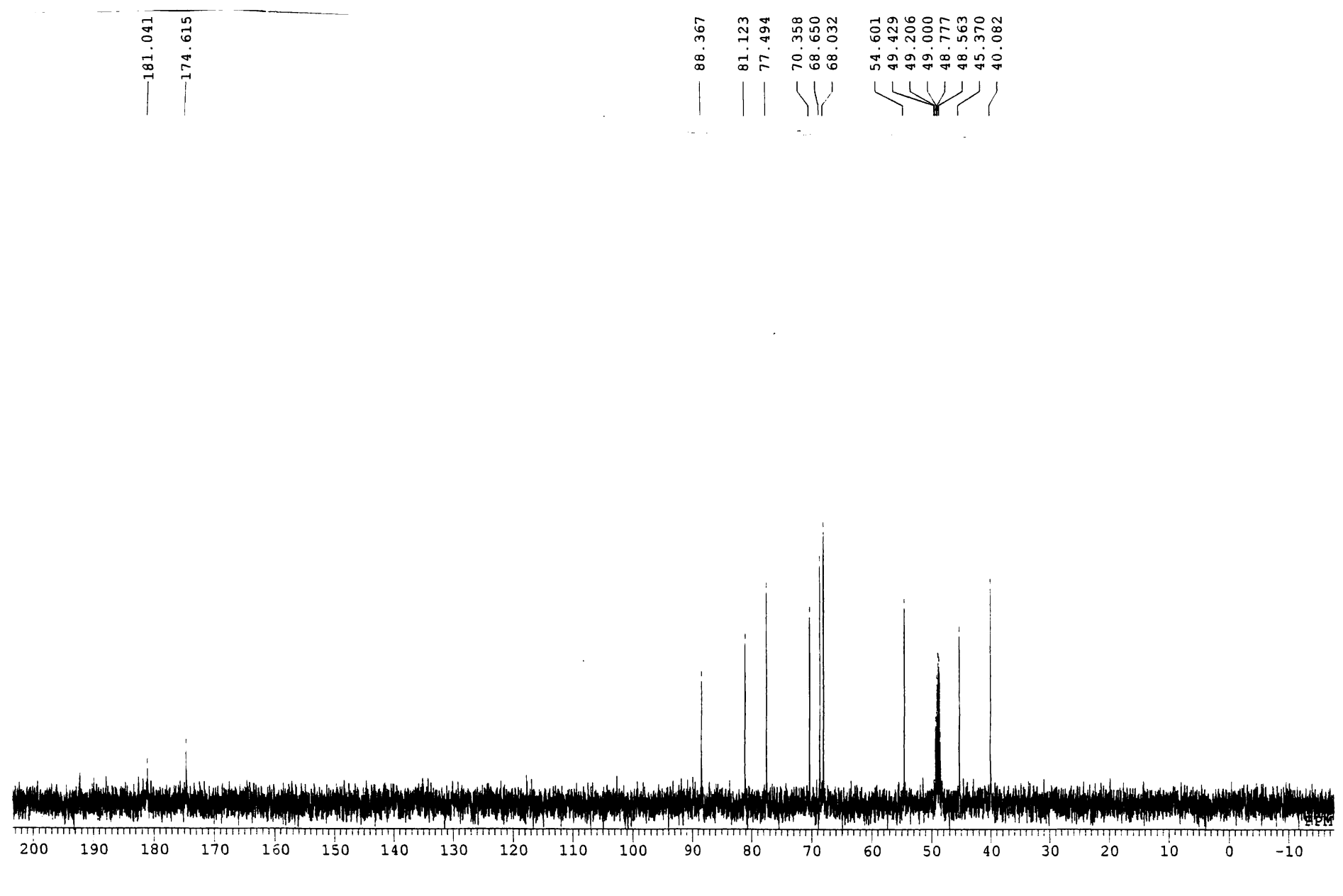